ARTICLE

https://doi.org/10.1038/s41467-019-10517-1

\title{
NCP activates chloroplast transcription by controlling phytochrome-dependent dual nuclear and plastidial switches
}

\author{
Emily J. Yang (10) 1,2,6,8, Chan Yul Yoo (1) 1,8, Jiangxin Liu" 3,4,8, He Wang ${ }^{1}$, Jun Cao ${ }^{5}$, Fay-Wei Li (i) 2,7, \\ Kathleen M. Pryer (1) ${ }^{2}$, Tai-ping Sun², Detlef Weigel ${ }^{5}$, Pei Zhou (1) ${ }^{3} \&$ Meng Chen (1) ${ }^{1}$
}

Phytochromes initiate chloroplast biogenesis by activating genes encoding the photosynthetic apparatus, including photosynthesis-associated plastid-encoded genes (PhAPGs). PhAPGs are transcribed by a bacterial-type RNA polymerase (PEP), but how phytochromes in the nucleus activate chloroplast gene expression remains enigmatic. We report here a forward genetic screen in Arabidopsis that identified NUCLEAR CONTROL OF PEP ACTIVITY (NCP) as a necessary component of phytochrome signaling for PhAPG activation. NCP is dual-targeted to plastids and the nucleus. While nuclear NCP mediates the degradation of two repressors of chloroplast biogenesis, PIF1 and PIF3, NCP in plastids promotes the assembly of the PEP complex for PhAPG transcription. NCP and its paralog RCB are non-catalytic thioredoxin-like proteins that diverged in seed plants to adopt nonredundant functions in phytochrome signaling. These results support a model in which phytochromes control PhAPG expression through light-dependent double nuclear and plastidial switches that are linked by evolutionarily conserved and dual-localized regulatory proteins.

\footnotetext{
${ }^{1}$ Department of Botany and Plant Sciences, Institute for Integrative Genome Biology, University of California, Riverside, CA 92521, USA. ${ }^{2}$ Department of Biology, Duke University, Durham, NC 27708, USA. ${ }^{3}$ Department of Biochemistry, Duke University Medical Center, Durham, NC 27710, USA. ${ }^{4}$ State Key Laboratory of Phytochemistry and Plant Resources in West China, Kunming Institute of Botany, Chinese Academy of Sciences, 650201 Kunming, China. ${ }^{5}$ Department of Molecular Biology, Max Planck Institute for Developmental Biology, 72076 Tübingen, Germany. ${ }^{6}$ Present address: Department of Pathology and Cell Biology, Columbia University Medical Center, New York, NY 10032, USA. ${ }^{7}$ Present address: Boyce Thompson Institute, Ithaca, NY 14853, USA.

${ }^{8}$ These authors contributed equally: Emily J. Yang, Chan Yul Yoo, Jiangxin Liu. Correspondence and requests for materials should be addressed to

P.Z. (email: peizhou@biochem.duke.edu) or to M.C. (email: meng.chen@ucr.edu)
} 
T he control of organellar gene expression in the mitochondria and plastids is critical for cellular reprogramming in the eukaryotic cell. The regulation of gene activity in plastids is particularly important for plants because, although the vast majority of the genetic material of the ancestral cyanobacterial endosymbiont has been transferred to the nucleus ${ }^{1}$, the plastid genome retains 100-120 genes encoding essential components of not only the plastidial transcriptional and translational machineries but also the photosynthetic apparatus ${ }^{2}$. The regulation of plastid-encoded photosynthesis-associated genes is pivotal for plants to establish photosynthetically active chloroplasts and thus is essential for plant survival.

Light is one of the most important environmental cues required for initiating chloroplast biogenesis in seed plants, including angiosperms (flowering plants) and some gymnosperms ${ }^{3,4}$. In dicotyledonous flowering plants, such as Arabidopsis thaliana, seedlings that germinate under the ground adopt a dark-grown developmental program called skotomorphogenesis, which promotes the elongation of the embryonic stem (hypocotyl) and inhibits leaf development and chloroplast biogenesis, a strategy that allows seedlings to emerge rapidly and easily from the soil. In darkness, the plastids in the leaf tissues differentiate into nongreen, photosynthetically inactive etioplasts. Emerging into the sunlight triggers seedlings to transition to photomorphogenesis, which attenuates hypocotyl elongation and stimulates leaf development. The photomorphogenetic developmental program also enables chloroplast biogenesis and photosynthesis ${ }^{5}$.

The transition to photomorphogenesis entails the massive transcriptional reprogramming of the nuclear genome initiated by photoreceptors, such as the red (R) and far-red (FR) photoreceptors, the phytochromes (PHYs), which play an essential role in chloroplast biogenesis ${ }^{6-8}$. The biological activity of PHYs can be turned on and off through light-dependent conformational switches between a R light-absorbing inactive Pr form and a FR light-absorbing active Pfr form 9 . In Arabidopsis, PHYs are encoded by five genes, PHYA-E, of which PHYA and PHYB are the predominant sensors of continuous FR and R light, respectively $y^{5,10}$. The earliest light response at the cellular level is the translocation of photoactivated PHYs from the cytoplasm to discrete subnuclear domains named photobodies ${ }^{11}$. PHYs bind directly to Phytochrome-Interacting Factors (PIFs) and colocalize with them on photobodies ${ }^{12,13}$. The PIFs are basic/helix-loophelix transcription factors antagonistic to photomorphogenesis ${ }^{14}$. Most PIFs accumulate to high levels in dark-grown seedlings to promote hypocotyl elongation and inhibit chloroplast biogenesis by activating growth-relevant genes and repressing nuclearencoded photosynthesis-associated genes, respectively ${ }^{15,16}$. PHYs repress the functions of PIFs by inhibiting their transcriptional activity and promoting their ubiquitin-and-proteasome-mediated degradation $13,14,17,18$. PHY-mediated PIF degradation is a central mechanism for inducing chloroplast biogenesis through the activation of photosynthesis-associated nuclear-encoded genes $(P h A N G s)^{14}$. The localization of PHYs to photobodies is closely associated with PIF3 degradation ${ }^{12,13,17,19,20}$.

Light also induces the transcription of photosynthesisassociated plastid-encoded genes (PhAPGs) ${ }^{21,22}$, which encode essential components of the photosynthetic apparatus, including the large subunit of the carbon fixation enzyme ribulose-1,5bisphosphate carboxylase/oxygenase (rbcL) and the photosystem II reaction center D1 protein (psbA) ${ }^{2}$. Plastidial genes are transcribed by two types of RNA polymerases: a phage-type nuclearencoded RNA polymerase (NEP) and a bacterial-type plastidencoded RNA polymerase (PEP) ${ }^{23}$. While the NEP preferentially transcribes housekeeping genes, including plastid ribosomal RNAs and the core subunits of the PEP, the PEP mainly transcribes PhAPGs ${ }^{24,25}$. How PHYs in the nucleus control PEP-mediated
PhAPG expression in plastids is largely unknown. A wellrecognized challenge has been the lack of an efficient forwardgenetic screening strategy that can distinguish chloroplastdeficient regulator mutants from other albino mutants with defects in genes encoding essential components of the chloroplast ${ }^{26}$. Our recent genetic studies of early PHY signaling have serendipitously uncovered a new class of photomorphogenetic mutants in Arabidopsis with a combination of albino and longhypocotyl seedling phenotypes ${ }^{19,27}$. The founding member of this new mutant class, hemera $(\mathrm{hmr})$, is defective in PHYB signaling and chloroplast biogenesis ${ }^{19,28}$. Albino mutants had been ignored previously in the context of light signaling because historically, chlorophyll-deficient mutants had been shown to retain normal PHY-mediated hypocotyl responses ${ }^{29,30}$. As a result, the entire class of tall-and-albino mutants like $h m r$ had been overlooked ${ }^{27}$. We hypothesized that some of the tall-and-albino mutants might define missing components of PHY signaling for activating PhAPG expression. To test this hypothesis, we performed a forward genetic screen for tall-and-albino mutants. This screen identified Nuclear Control of PEP Activity (NCP), a dual-targeted nuclear/plastidial protein required for both the nuclear and plastidial signaling steps of $P h A P G$ activation. We present evidence that NCP and its Arabidopsis paralog diverged in seed plants to adopt distinct roles in PHY signaling for PhAPG activation. We propose that PHYs control plastidial PhAPG expression via nucleus-to-plastid signaling, which comprises light-dependent double nuclear and plastidial switches that are governed by evolutionarily conserved and dual-localized regulatory proteins.

\section{Results}

Identification of NCP. We performed a forward genetic screen in continuous monochromatic $\mathrm{R}$ light for mutants with a combination of tall and albino seedling phenotypes. The screen was conducted in the $P B G$ (PHYB-GFP) background, a transgenic line in the null phyB-5 background complemented with functional PHYB-GFP 31 . This screening strategy allowed us to assess whether the early signaling event of photobody formation is impaired in the mutants ${ }^{19}$. From 2,000 N-ethyl-N-nitrosourea or ethyl methanesulfonate mutagenized $\mathrm{F}_{2} P B G$ families, we identified 23 tall-and-albino mutants. In this study, we focused on one of the mutants, which we named ncp-1 (nuclear control of PEP activity-1) (Fig. 1a, b).

We used SHOREmap and mapped the mutation co-segregating with the tall-and-albino phenotype in $n c p-1 / P B G$ to a single G-toA mutation in chromosome 2 at nucleotide $13,538,458$, which results in a premature stop codon in gene At2g31840 (Fig. 1c). Expressing the predicted cDNA of At2g31840 under the constitutive ubiquitin-10 promoter complemented the tall-andalbino phenotype of $n c p-1 / P B G$ (Supplementary Fig. 1a, b). We identified a second $n c p$ allele in the Col-0 background, $n c p-10$, which carries a T-DNA insertion after nucleotide $13,538,811$ in the second exon of At2g31840 (Fig. 1c). The mRNA levels of NCP in $n c p-1 / P B G$ and $n c p-10$ were more than 11 -fold lower than those in their respective parental lines (Supplementary Fig. 1c). Both mutants are likely null alleles. Similar to $n c p-1 / P B G, n c p-10$ was tall and albino (Fig. 1d, e). Together, these results demonstrate that At2g31840 is NCP.

$N C P$ encodes a 350-amino-acid protein with a few recognizable motifs (Fig. 1c). Analysis by Phyre2 software (www.sbg.bio.ic.ac. $\mathrm{uk} /$ phyre2/) revealed a thioredoxin (Trx)-like domain (amino acid 212-319) at its C-terminus ${ }^{32}$. Interestingly, two subcellular targeting signals were found in NCP: an N-terminal transit peptide (amino acids 1-48) predicted by ChloroP $^{33}$ for chloroplast import and a nuclear localization signal (NLS) detected by NLS mapper ${ }^{34}$ between amino acids 118 and 145 

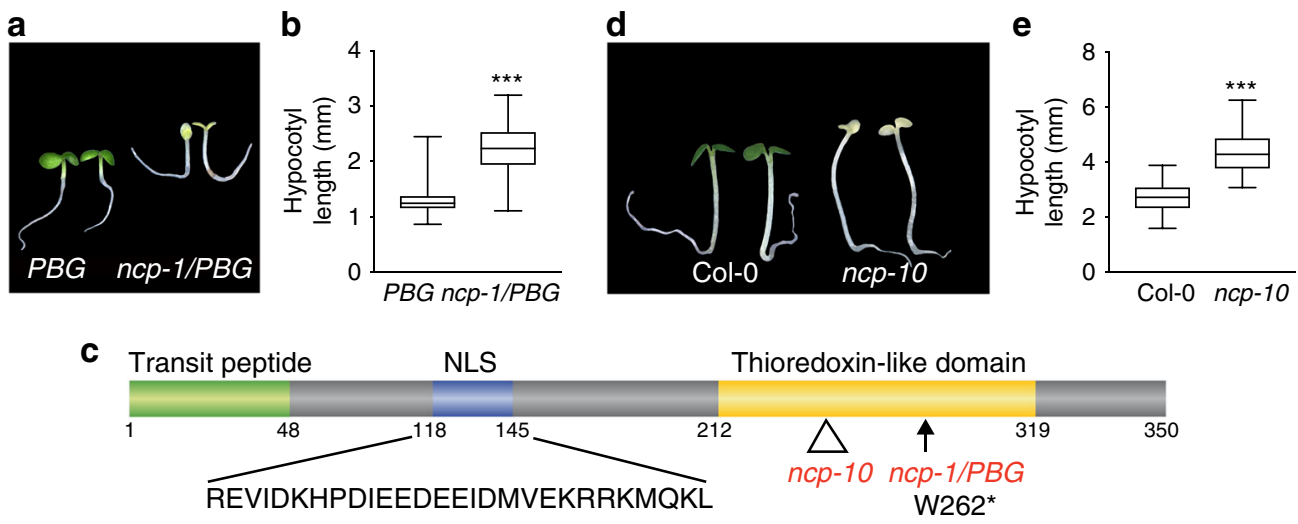

$\mathbf{f}$
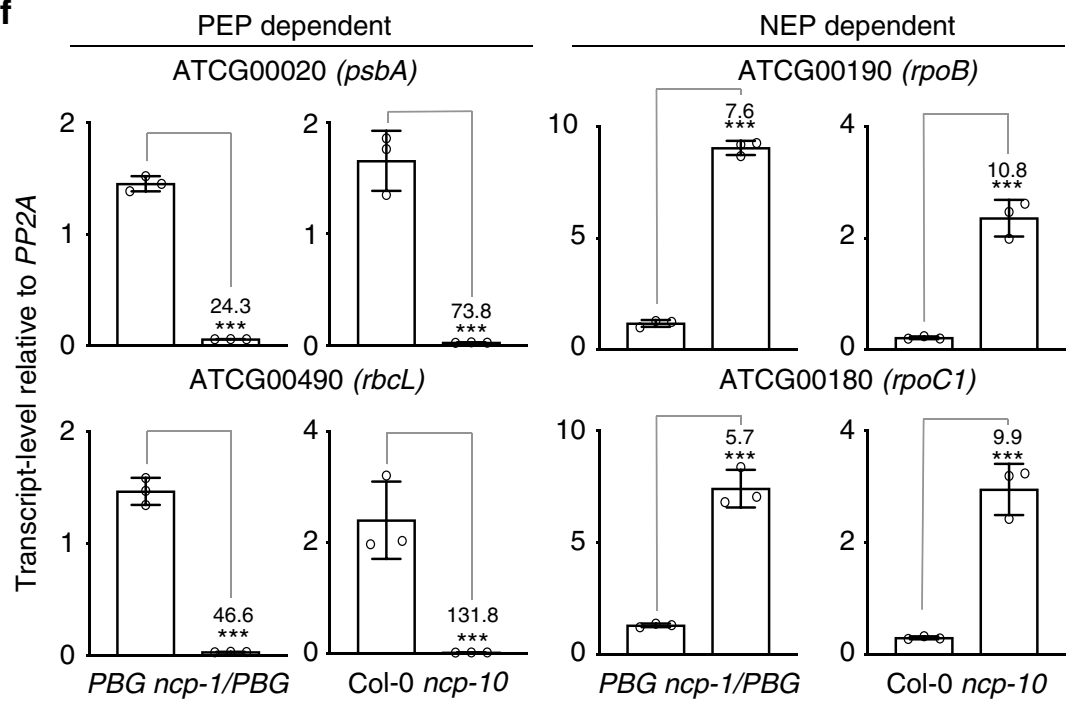

ATCG00180 (rpoC1)

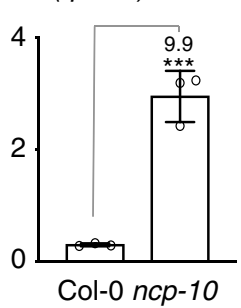

Fig. 1 Identification of NCP by a screen for tall-and-albino mutants. a Representative images of 4-day-old PBG and ncp-1/PBG seedlings grown in $10 \mu \mathrm{mol} \mathrm{m} \mathrm{m}^{-2} \mathrm{~s}^{-1}$ continuous R light. b Box-and-whisker plots showing hypocotyl measurements of the seedlings in a. c Schematic illustration of the predicted domain structure of NCP. The mutation in ncp-1/PBG and the T-DNA insertion site in ncp-10 are indicated. NLS, nuclear localization signal.

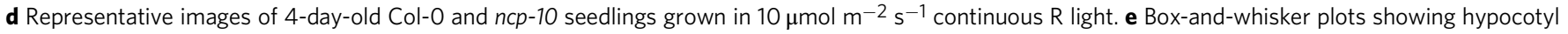
measurements of the seedlings in $\mathbf{d}$. $\mathbf{f}$ qRT-PCR results showing the steady-state mRNA levels of the PEP-dependent psbA and $r b c L$ and the NEP-dependent $r p o B$ and rpoC1 in 4-day-old PBG, ncp-1/PBG, Col-0, and ncp-10 seedlings grown in $10 \mu m o l ~ \mathrm{~m}^{-2} \mathrm{~s}^{-1}$ continuous $\mathrm{R}$ light. Error bars represent SD of three biological replicates. The transcript levels were calculated relative to those of $P P 2 A$. The numbers above the right columns are the fold changes in gene expression between the columns. For the box-and-whisker plots in $\mathbf{b}$ and $\mathbf{e}$, the boxes represent from the 25 th to the 75 th percentiles, and the bars equal the median values. For $\mathbf{b}, \mathbf{e}$, and $\mathbf{f}$, asterisks ( $\left.{ }^{\star \star \star}\right)$ indicate a statistically significant difference between the values of the mutants and those of the wild-type or the parental line (Student's $t$-test, $p \leq 0.001$ ). The source data of the hypocotyl measurements in $\mathbf{b}$, e and the qRT-PCR data in $\mathbf{f}$ are provided in the Source Data file

(Fig. 1c). NCP has been identified previously as MRL7-L (Mesophyll-cell RNAi Library line 7-like) ${ }^{35}$ and SVR4-like (Suppressor of Variegation4-like) ${ }^{36}$ because of its essential role in chloroplast biogenesis, particularly for $P h A P G$ activation $^{35,36}$. However, the precise function of NCP in PhAPG regulation is still unknown. In agreement with published results, the expression of two PEP-dependent PhAPGs, $p s b A$ and $r b c L$, was downregulated by more than 24 -fold and 73 -fold in $n c p-1 / P B G$ and $n c p-10$, respectively, whereas the expression of NEP-dependent genes, such as $r p o B$ and $r p o C 1$, was upregulated by 5.7 -fold to 10.8 -fold (Fig. 1f). The divergent effects on PEP- and NEP-regulated genes are characteristics of mutants impaired specifically in the PEP function 24,28 .

NCP mediates phytochrome signaling. To investigate the role of NCP in PHY signaling, we analyzed the hypocotyl elongation responses of the ncp mutants in continuous FR and $\mathrm{R}$ light to assess their effectiveness in PHYA and PHYB signaling, respectively $^{37}$. These experiments showed that $n c p-10$ and $n c p-1 / P B G$ were hyposensitive to $\mathrm{R}$ and FR light (Fig. 2a-d). The long hypocotyl phenotype of $n c p$ relies on PHY signaling, as $n c p-10 /$ phyB-9 and ncp-10/phyA-211 double mutants were not taller than phyB-9 and phyA-211, respectively (Fig. 2e-h). To further demonstrate NCP's role in PHY signaling, we crossed $n c p-1$ to a constitutively active $p h y B$ allele $Y H B$, which carries a $Y 276 \mathrm{H}$ mutation in PHYB's photosensory chromophore attachment domain that locks PHYB in an active form ${ }^{38}$. In the $n c p-1 / Y H B$ double mutant, the constitutive photomorphogenetic phenotypes of $Y H B$ in the dark were partially suppressed (Fig. 2i, j), confirming that NCP is required for PHYB signaling. In contrast to the defects in response to FR and R light, $n c p-10$ and $n c p-1 / P B G$ had normal hypocotyl responses in white and blue light (Supplementary Fig. 2), suggesting that NCP is not required for blue light signaling by cryptochromes. Together, these genetic data indicate that NCP participates specifically in PHY signaling. 

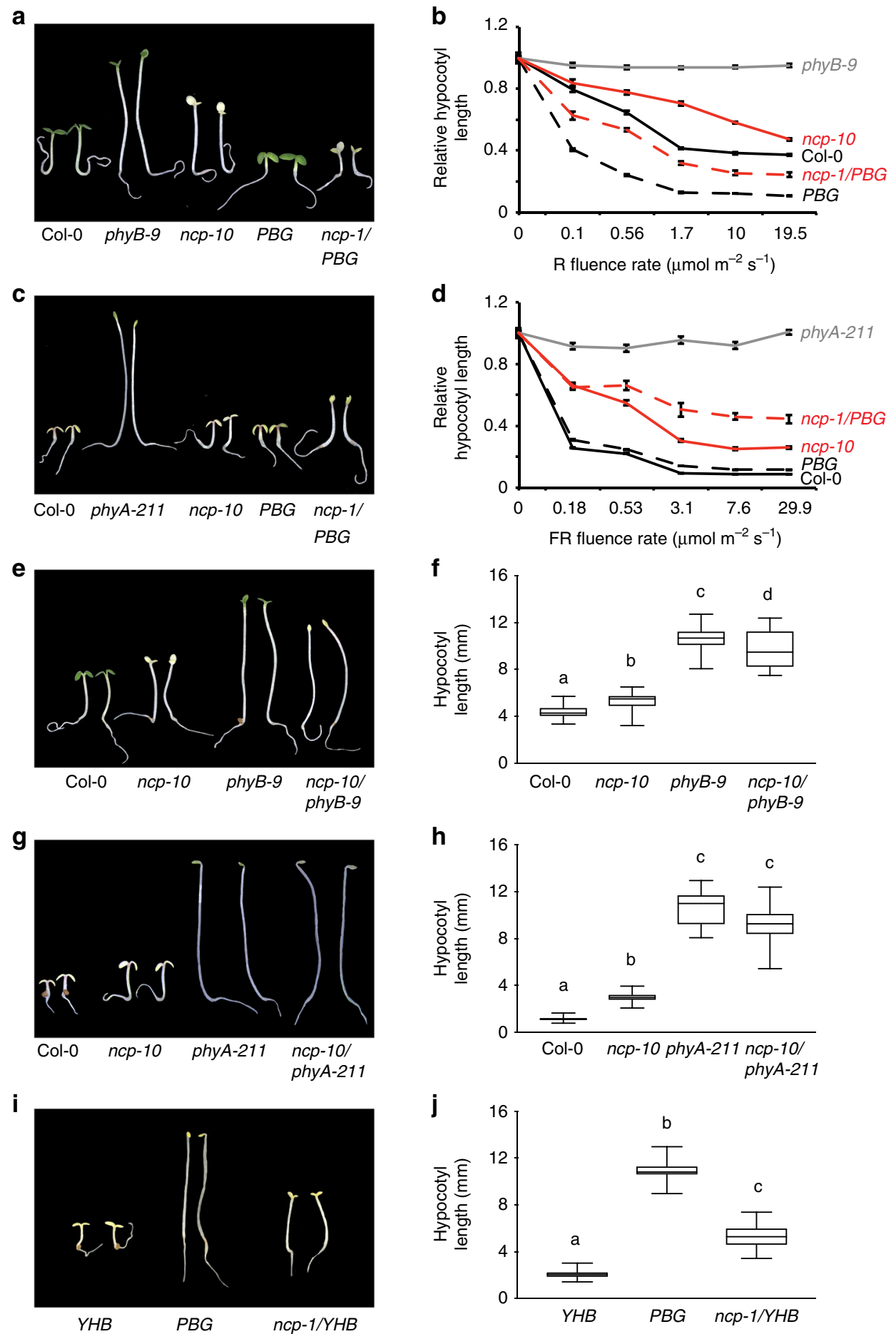

Fig. 2 NCP mediates phytochrome signaling. a Representative images of 4-day-old Col-0, phyB-9, ncp-10, PBG, and ncp-1/PBG seedlings grown in $10 \mu \mathrm{mol} \mathrm{m} \mathrm{m}^{-2} \mathrm{~s}^{-1} \mathrm{R}$ light. $\mathbf{b} \mathrm{R}$ light fluence response curves showing the hypocotyl elongation responses of 4-day-old Col-0 (solid black line), phyB-9 (solid gray line), ncp-10 (solid red line), PBG (dotted black line), and ncp-1/PBG (dotted red line) seedlings grown in the dark and a series of $\mathrm{R}$ light intensities. c Representative images of 4-day-old Col-0, phyA-211, ncp-10, PBG, and ncp-1/PBG seedlings grown in $10 \mu \mathrm{mol} \mathrm{m}^{-2} \mathrm{~s}^{-1} \mathrm{FR}$ light. $\mathbf{d}$ Fluence response curves for FR light showing the relative hypocotyl lengths of 4-day-old Col-0 (solid black line), phyA-211 (solid gray line), ncp-10 (solid red line), PBG (dotted black line) and ncp-1/PBG (dotted red line) seedlings grown in the dark and a series of FR light intensities. For $\mathbf{b}$ and $\mathbf{d}$, error bars represent $\mathrm{SE}$, and hypocotyl length in the light was calculated relative to that in the dark. e, Representative images of 4-day-old Col-0, ncp-10, phyB-9, and ncp-10/phyB-9 seedlings grown in $10 \mu \mathrm{mol} \mathrm{m} \mathrm{m}^{-2} \mathrm{~s}^{-1} \mathrm{R}$ light. $\mathbf{f}$ Box-and-whisker plots showing hypocotyl length measurements of the seedlings in e. $\mathbf{g}$ Representative images of 4 day-old Col-0, ncp-10, phyA-211, and ncp-10/phyA-211 seedlings grown in $10 \mu \mathrm{mol} \mathrm{m}^{-2} \mathrm{~s}^{-1} \mathrm{FR}$ light. h Box-and-whisker plots showing hypocotyl length measurements of the seedlings in $\mathbf{g}$. i Representative images of 4-day-old dark-grown YHB, PBG, and ncp-1/YHB seedlings. $\mathbf{j}$ Box-and-whisker plots showing hypocotyl length measurements of the seedlings in $\mathbf{i}$. For the box-and-whisker plots in $\mathbf{f}, \mathbf{h}$, and $\mathbf{j}$, the boxes represent from the 25 th to the 75 th percentiles, and the bars equal the median values; samples with different letters show statistically significant differences in hypocotyl length (ANOVA, Tukey's HSD, $p \leq 0.001, n>28$ ). The source data of the hypocotyl measurements in $\mathbf{b}, \mathbf{d}, \mathbf{f}, \mathbf{h}$, and $\mathbf{j}$ are provided in the Source Data file 
a

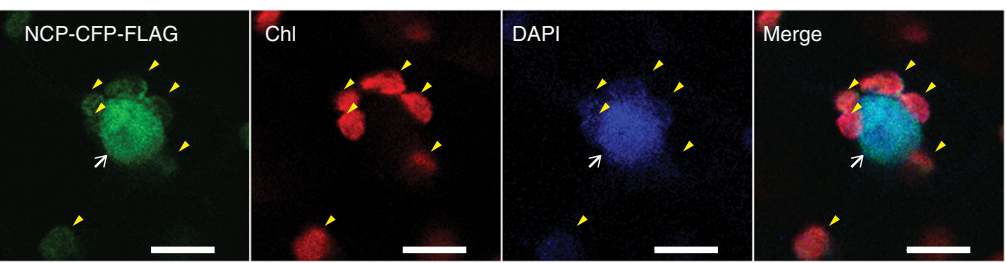

b
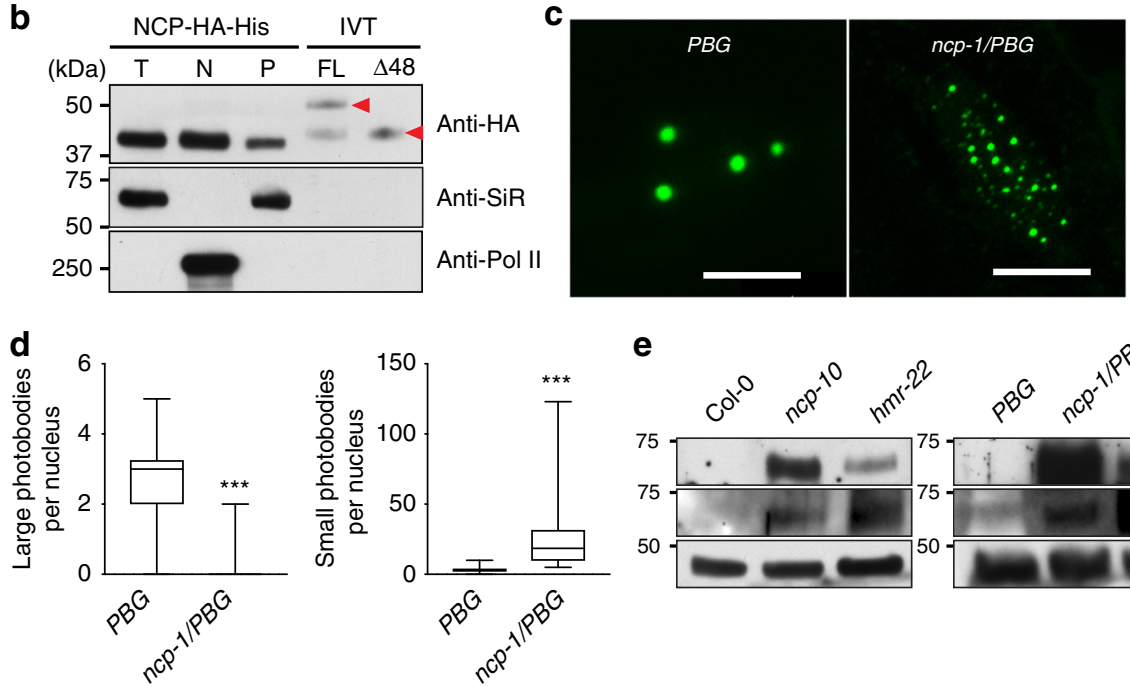

e

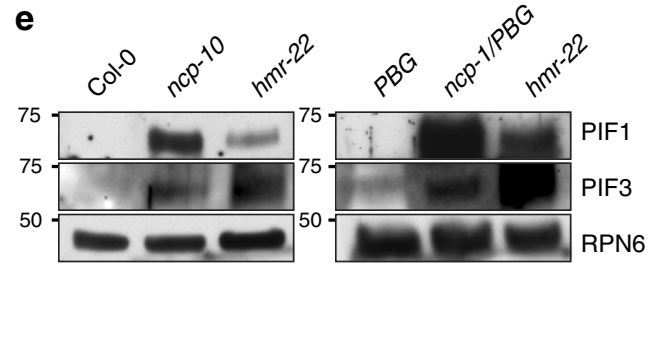

Fig. 3 NCP participates in photobody biogenesis and PIF degradation in the nucleus. a Confocal images showing the subcellular localization pattern of transiently expressed NCP-CFP-FLAG in tobacco leaf cells. NCP-CFP-FLAG signals (green) were detected in chloroplasts (marked by yellow arrowheads) and the nucleus (indicated by a white arrow). The nucleus was labeled with DAPI. Chloroplasts were labeled with DAPI in the blue channel, as well as by chlorophyll autofluorescence in the red channel. Scale bars represent $10 \mu \mathrm{m}$. b Immunoblots showing NCP-HA-His from total, (T), nuclear (N), and plastidial (P) protein fractions of 2-day-old NCP-HA-His/ncp-10 seedlings grown in $10 \mu \mathrm{mol} \mathrm{m} \mathrm{m}^{-2} \mathrm{~s}^{-1} \mathrm{R}$ light. In vitro translated (IVT) NCP-HA-His and $\mathrm{NCP} \triangle 48-\mathrm{HA}$-His (indicated by red arrowheads) were used as molecular size controls. The HA-tagged NCP proteins were detected via anti-HA antibodies. Ferredoxin:sulfite reductase ( $\mathrm{SiR}$ ) and RNA Pol II were used as controls for the plastidial and nuclear fractions, respectively. c Confocal images of PHYBGFP photobodies in epidermal cells from the top one third of the hypocotyls of 4-day-old PBG and ncp-1/PBG seedlings grown in $10 \mu \mathrm{mol} \mathrm{m}^{-2} \mathrm{~s}^{-1} \mathrm{R}$ light. Scale bars represent $5 \mu \mathrm{m}$. d Box-and-whisker plots showing the numbers of large $\left(>0.73 \mu \mathrm{m}^{3}\right.$, left panel) and small $\left(<0.73 \mu \mathrm{m}^{3}\right.$, right panel) photobodies per nucleus in the epidermal cells of the top one third of the hypocotyls of $P B G$ and ncp-1/PBG seedlings. The boxes represent from the 25th to the 75th percentiles, the whiskers extend to the maximum and minimum data points, and the center lines in each box indicate the median value. Asterisks ( ${ }^{\star \star \star}$ ) indicate a statistically significant difference between the value in $n c p-1 / P B G$ and that in PBG (Student's $t$-test, $p \leq 0.001$ ). e Immunoblots showing the PIF1 and PIF3 levels in 4-day-old Col-0, ncp-10, hmr-22, PBG, and ncp-1/PBG seedlings grown in $10 \mu \mathrm{mol} \mathrm{m} \mathrm{m}^{-2} \mathrm{~s}^{-1} \mathrm{R}$ light. RPN6 was used as a loading control. The source data for immunoblots in $\mathbf{b}$, e and photobody analysis in $\mathbf{d}$ are provided in Source Data file

NCP is a dual-targeted phytochrome signaling component. The result that NCP participates in PHYA signaling and PHYB signaling, which occur mainly in the nucleus ${ }^{39,40}$, contradicts the published data suggesting that NCP localizes only to plastids ${ }^{35}$. We therefore asked whether NCP, with a predicted NLS (Fig. 1c), is also targeted to the nucleus. We found that transiently expressed NCP tagged with CFP and FLAG (RCB-CFP-FLAG) in tobacco cells was dispersed in both chloroplasts and the nucleus (Fig. 3a). In agreement with this result, a functional HA-tagged and His-tagged NCP expressed in ncp-10 (NCP-HA-His/ncp-10) was detected in the nuclear and plastidial protein fractions (Fig. 3b and Supplementary Fig. 3). Surprisingly, although plastidial NCP is expected to be smaller than nuclear NCP due to the removal of its transit peptide during plastid import, the total, nuclear, and plastidial fractions of NCP-HA-His had similar molecular masses (Fig. 3b). To further examine the size of NCPHA-His, we ran side-by-side in vitro translated full-length NCP$\mathrm{HA}-\mathrm{His}$ and the predicted plastidial NCP without the N-terminal 48 amino acids (NCP $\triangle 48-\mathrm{HA}$-His). NCP-HA-His in vivo was significantly smaller than the in vitro translated full-length NCPHA-His and similar to NCP $\triangle 48-\mathrm{HA}-\mathrm{His}$ (Fig. 3b). These results indicate that NCP is dual-targeted to plastids and the nucleus and imply that NCP might localize to the plastids first and then translocate to the nucleus similar to $\mathrm{HMR}^{41}$.

To understand how NCP participates in PHY signaling, we asked whether NCP is required for the earliest light response, PHYB localization to photobodies ${ }^{11}$. In $P B G$ seedlings grown under $10 \mu \mathrm{mol} \mathrm{m} \mathrm{m}^{-2} \mathrm{~s}^{-1} \mathrm{R}$ light, PHYB-GFP localized to a few large photobodies (Fig. 3c, d) ${ }^{19,42}$. In striking contrast, PHYB-GFP localized mostly to small photobodies in $n c p-1 / P B G$ (Fig. 3c, d). We then tested if NCP is required for the PHYmediated degradation of the antagonistic transcription factors of PHY signaling, the PIFs, because PIF degradation is closely associated with PHYB localization to large photosbodies ${ }^{19,42}$. Intriguingly, the two well-characterized light-labile PIFs, PIF1, and PIF3 ${ }^{14}$, accumulated or failed to be completely degraded in light-grown $n c p-1 / P B G$ and $n c p-10$ (Fig. 3e). Together, these results demonstrate that $\mathrm{NCP}$ participates in the early light signaling events of photobody biogenesis and the degradation of PIF1 and PIF3.

NCP activates PhAPGs in the nucleus and plastids. The PEP forms multisubunit protein complexes consisting of the bacterial- 
type core subunits and plant-specific PEP-associated proteins $^{28,43}$. We have shown recently that PhAPGs are activated by a linked nuclear and plastidial dual-switching mechanism in which PHY-mediated PIF degradation in the nucleus triggers the assembly of the PEP into a $1000-\mathrm{kDa}$ complex in plastids for $P h A P G$ transcription ${ }^{44}$. With four PIF genes knocked out, PIF1, PIF3, PIF4, and PIF5, the pifq mutant could trigger PEP assembly and $P h A P G$ activation in the dark ${ }^{44}$. The dual nuclear-andplastidial localization of NCP raised the question of whether NCP only functions in promoting PIF degradation in the nucleus or also regulates PEP assembly and activation directly in plastids. To distinguish between these two possibilities, we generated a $n c p$ 10/pif1/pif3/pif4/pif5 (ncp-10/pifq) quintuple mutant. We reasoned that if NCP activates PhAPG expression mainly by promoting PIF degradation in the nucleus, removing the four PIFs in $n c p-10$ should rescue its albino phenotype. The ncp-10/pifq mutant largely rescued the long-hypocotyl phenotype of $n c p-10$ (Fig. 4a). However, the $n c p-10 / p i f q$ mutant was slightly but significantly taller than pifq (Fig. 4a, b), which could be due to NCPdependent regulation of other PIFs or a PIF-independent retrograde signaling from the defective chloroplasts, as ncp-10/pifq remained albino ${ }^{45}$. The expression of PEP-dependent PhAPGs was still impaired in $n c p-10 / p i f q$, while the expression of NEPdependent plastidial genes was elevated (Fig. 4c). We resolved the PEP complex from Arabidopsis using blue-native gel electrophoresis and monitored its size by immunoblotting using antibodies against either the core $\beta$ subunit, rpoB, or one of the PEPassociated proteins, HMR/pTAC12 $19,28,43$. We found that the PEP failed to form a $1000-\mathrm{kDa}$ complex in $n c p-10$ (Fig. $4 \mathrm{~d}$ ), indicating that NCP is required for PEP assembly. The defect in PEP assembly was not rescued in ncp-10/pifq (Fig. 4d). Together, these results indicate that in addition to its nuclear function in PIF degradation, NCP also facilitates PEP assembly directly in plastids.

NCP and its paralog $R C B$ diverged in seed plants. NCP has a paralog in Arabidopsis, At4g28590, which we named Regulator of Chloroplast Biogenesis $(R C B)^{44}$. Previous studies have shown that $N C P$ and $R C B$ are present in angiosperms ${ }^{35}$. However, only one copy of NCP-like gene was found in the genomes of nonflowering plants, such as the moss Physcomitrella patens and lycophyte Selaginella moellendorffi ${ }^{35,46}$. Because these analyses did not include other seed plants, like gymnosperms, or the lineages between seed plants and lycophytes, like ferns, it remains unclear when NCP and RCB diverged during the evolution of land plants. We therefore searched for NCP-like sequences in all major land plant lineages, including bryophytes, lycophytes, ferns, and seed plants, and utilized Randomized Axelerated Maximum Likelihood (RAxML) to construct a phylogenetic tree with bootstrapping 47 . The resulting phylogeny revealed a single copy of NCP-like genes in ferns, lycophytes, and bryophytes, whereas the seed plants, including both angiosperms and gymnosperms, contain NCP and RCB (Fig. 5a). NCP-like sequences have not been identified in prokaryotic photosynthetic organisms, such as Rhodobacter sphaeroides and Synechocystis sp. PCC 6803, or in the single-cell green alga Chlamydomonas reinhardtii ${ }^{35,46}$. Consistent with these results, we did not find NCP homologs in the algal genomes of Klebsormidium flaccidum ${ }^{48}$ and Micromonas pusilla CCMP1545 ${ }^{49}$. These results indicate that an NCP-like gene emerged in early land plants and duplicated and diverged into $N C P$ and $R C B$ in seed plants.

Intriguingly, we also identified RCB from the screen for talland-albino mutants ${ }^{44}$. We showed that RCB is dual-localized to the plastids and the nucleus ${ }^{44}$. However, different from NCP, plastidal RCB does not play an essential role in chloroplast biogenesis $^{44}$. Instead, RCB initiates chloroplast biogenesis primarily in the nucleus to promote PIF1 and PIF3 degradation (Fig. 5d $)^{44}$. These results indicate that NCP and RCB have adopted non-redundant roles in regulating chloroplast biogenesis. The $n c p-10 / r c b-10$ double mutant had long hypocotyl and albino phenotypes that were similar to those of the single mutants (Fig. 5b, c), suggesting that NCP and RCB function in the same PHY-dependent pathway for initiating chloroplast biogenesis. We propose that $N C P$ and $R C B$ diverged to evolve distinct regulatory roles in PHY signaling likely to accommodate the regulation of chloroplast biogenesis by light in seed plants.

NCP and RCB contain a non-catalytic thioredoxin-like domain. NCP and RCB possess a C-terminal Trx-like domain. Trx is a small redox-active protein with a universally conserved dithiol (-Cys-X-X-Cys-) active site in which the Cys residues provide the sulfhydryl groups required for reducing disulfide bonds in target proteins ${ }^{50}$. Interestingly, neither NCP nor RCB contains the -Cys-X-X-Cys- catalytic motif (Fig. 6a). Surprisingly, a previous study suggested that RCB had Trx activity in vitro ${ }^{51}$. To seek a structural explanation for the Trx reductase activity, we solved the NMR structure of NCP's Trx-like domain. The Trxlike domain of NCP displays a typical Trx-like fold-a fivestranded $\beta$-sheet with a $\beta 1-\beta 5$ arrangement surrounded by four $\alpha$ helixes (Fig. 6b and Supplementary Figs. 4-6). A stereo view of the NMR structural ensemble of the NCP Trx-like domain is shown in Supplementary Fig. 7, and the detailed statistics on the structural ensemble are given in Table 1. Based on the structure of NCP, we modeled the structure of the Trx-like fold of RCB (Fig. 6c). The structure of NCP's Trx-like domain overlays nicely with that of E. coli Trx (Fig. 6d) ${ }^{52}$. The Trx-like domains of NCP and RCB exhibit the same $\beta \alpha \beta \alpha \beta \alpha \beta \beta \alpha$ secondary structural arrangement as those of $E$. coli Trx (Fig. 6a-d) but without a -Cys-X-X-Cys- catalytic motif. Therefore, the structural data do not support a Trx reductase activity. We then performed the insulin reduction assay using $E$. coli expressed recombinant NCP and RCB fragments (Fig. 6e) ${ }^{53}$. These experiments did not detect any Trx reductase activity in NCP or RCB (Fig. 6f, g). Based on the structural and biochemical analyses, we conclude that RCB and NCP contain a non-catalytic Trx-like domain.

\section{Discussion}

PHY signaling initiates chloroplast biogenesis by activating photosynthesis-associated genes encoded by the nuclear and plastidial genomes. PHYs regulate nuclear gene expression by directly modulating the activity and stability of transcription factors in the nucleus ${ }^{14,27}$. However, how PHYs in the nucleus control gene expression in plastids, particularly the activation of PhAPGs by the PEP, remains poorly understood. Here we report the identification of a dual-targeted nuclear/plastidial signaling component, NCP, which participates in both nuclear and plastidial PHY signaling events for PhAPG activation (Fig. 5d). Our results provide evidence supporting the model that PHYs control plastidial gene expression through dual nuclear and plastidial switches, which are governed by evolutionarily conserved dualtargeted regulatory proteins (Fig. 5d).

It has been proposed for decades that plastid-encoded genes are controlled by the cell nucleus through anterograde nucleusto-plastid signaling ${ }^{26}$. A well-recognized challenge, which hindered the identification of such an anterograde signaling pathway, had been the lack of an effective forward-genetic screening strategy for distinguishing chloroplast-deficient regulator mutants from other albino mutants with defects in genes encoding essential components of the chloroplast ${ }^{26}$. Different from all previous genetic screens, we searched for mutants with a 
a

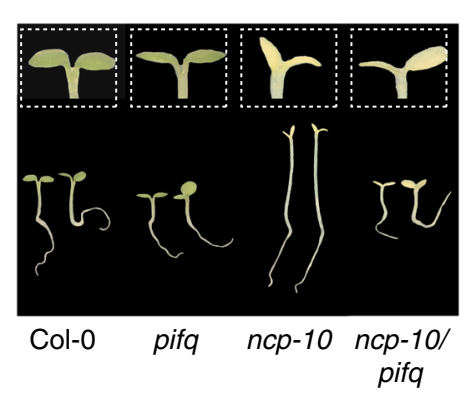

C

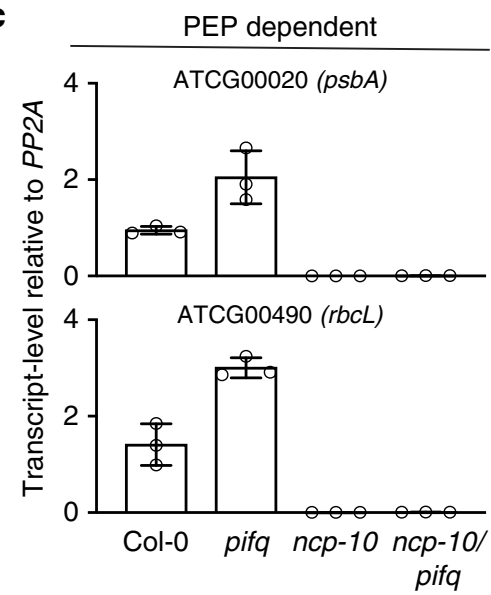

d

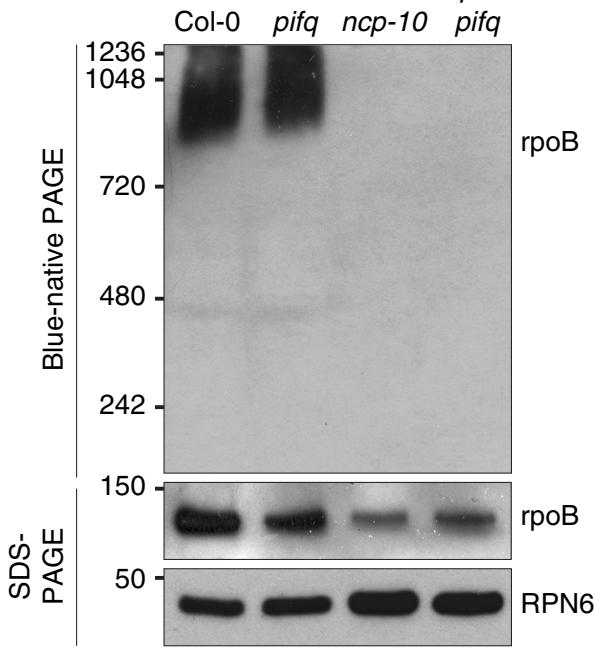

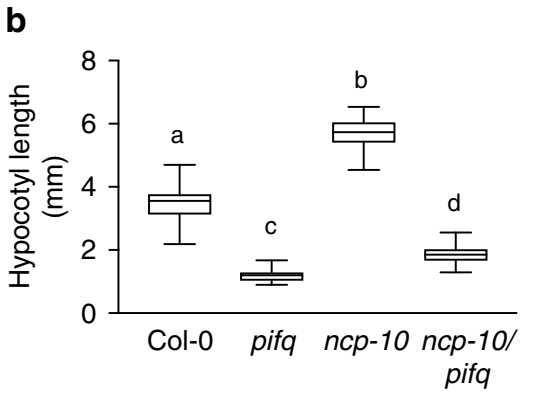
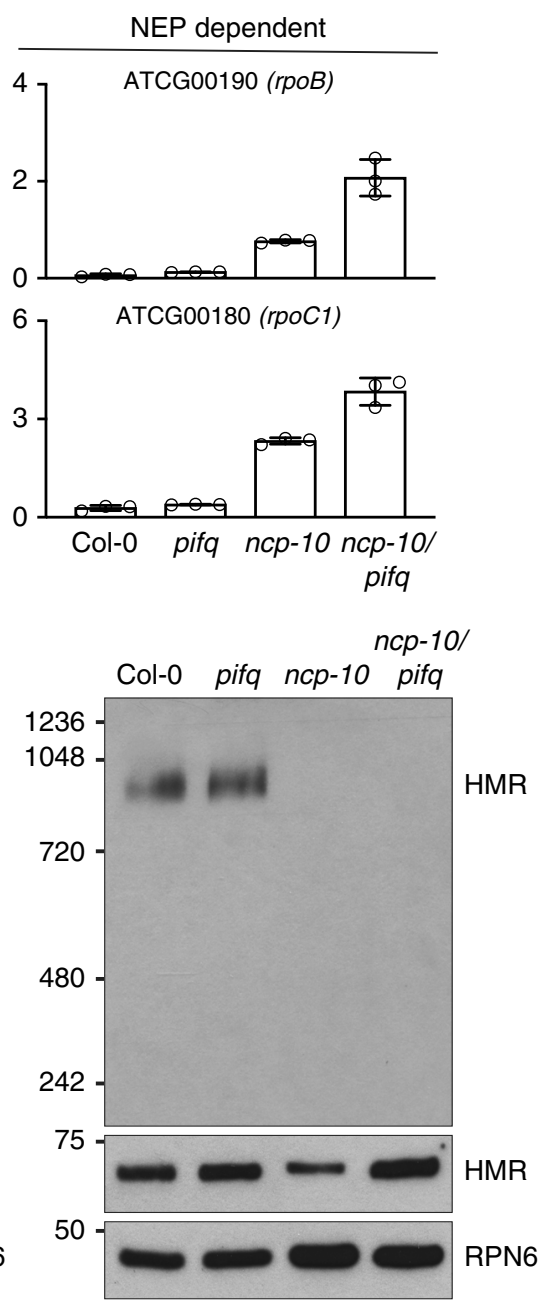

Fig. 4 NCP promotes the assembly and activation of the PEP in plastids. a A ncp-10/pifq mutant rescues the long hypocotyl phenotype, but not the albino phenotype, of ncp-10. Left panel: representative images of 4-day-old Col-0, pifq, ncp-10, and ncp-10/pifq seedlings grown in $10 \mu \mathrm{mol} \mathrm{m}^{-2} \mathrm{~s}^{-1} \mathrm{R}$ light. Inlets show magnified images of embryonic leaves of one of the corresponding seedlings below. $\mathbf{b}$ Box-and-whisker plots showing hypocotyl length measurements for the seedlings in $\mathbf{a}$. The boxes represent from the 25th to the 75th percentiles, and the bars represent the median values. Samples with different letters have statistically significant differences in hypocotyl length (ANOVA, Tukey's HSD, $p \leq 0.001, n>30$ ). c qRT-PCR analyses of the transcript levels of selected PEP-dependent and NEP-dependent genes in 4-day-old Col-0, pifq, ncp-10, and ncp-10/pifq seedlings grown under $10 \mu \mathrm{mol} \mathrm{m}^{-2} \mathrm{~s}^{-1} \mathrm{R}$ light.

Transcript levels were calculated relative to those of $P P 2 A$. Error bars represent SD of three biological replicates. $\mathbf{d}$ Immunoblots showing the level of the PEP complex (blue-native PAGE), as well as the levels of rpoB and HMR proteins (SDS-PAGE) in 4-day-old Col-0, pifa, ncp-10, and ncp-10/pifq seedlings grown in $10 \mu \mathrm{mol} \mathrm{m} \mathrm{m}^{-2} \mathrm{~s}^{-1} \mathrm{R}$ light using antibodies against rpoB or HMR. RPN6 was used as a loading control. The source data underlying the hypocotyl measurements in $\mathbf{b}$, the qRT-PCR data in $\mathbf{c}$, and the immunoblots in $\mathbf{d}$ are provided in the Source Data file

combination of long hypocotyl and albino phenotypes, which is indicative of defects in nuclear PHY signaling and chloroplast biogenesis ${ }^{17,19}$. This forward genetic screen identified NCP and its paralog, $\mathrm{RCB}^{44}$. Our investigation of RCB revealed that PHYs induce $P h A P G$ transcription through a nucleus-to-plastid anterograde signaling pathway linking two required switching mechanisms: PHY-mediated PIF degradation in the nucleus and the assembly of the PEP into a $1000-\mathrm{kDa}$ complex in plastids (Fig. 5d ${ }^{44}$. Interestingly, although RCB is dual-targeted to plastids and the nucleus, it activates $P h A P G$ expression primarily in the nucleus by promoting PIF1 and PIF3 degradation ${ }^{44}$. An $r c b$ 10/pifq mutant, in which four nuclear PIF transcription factors 
a
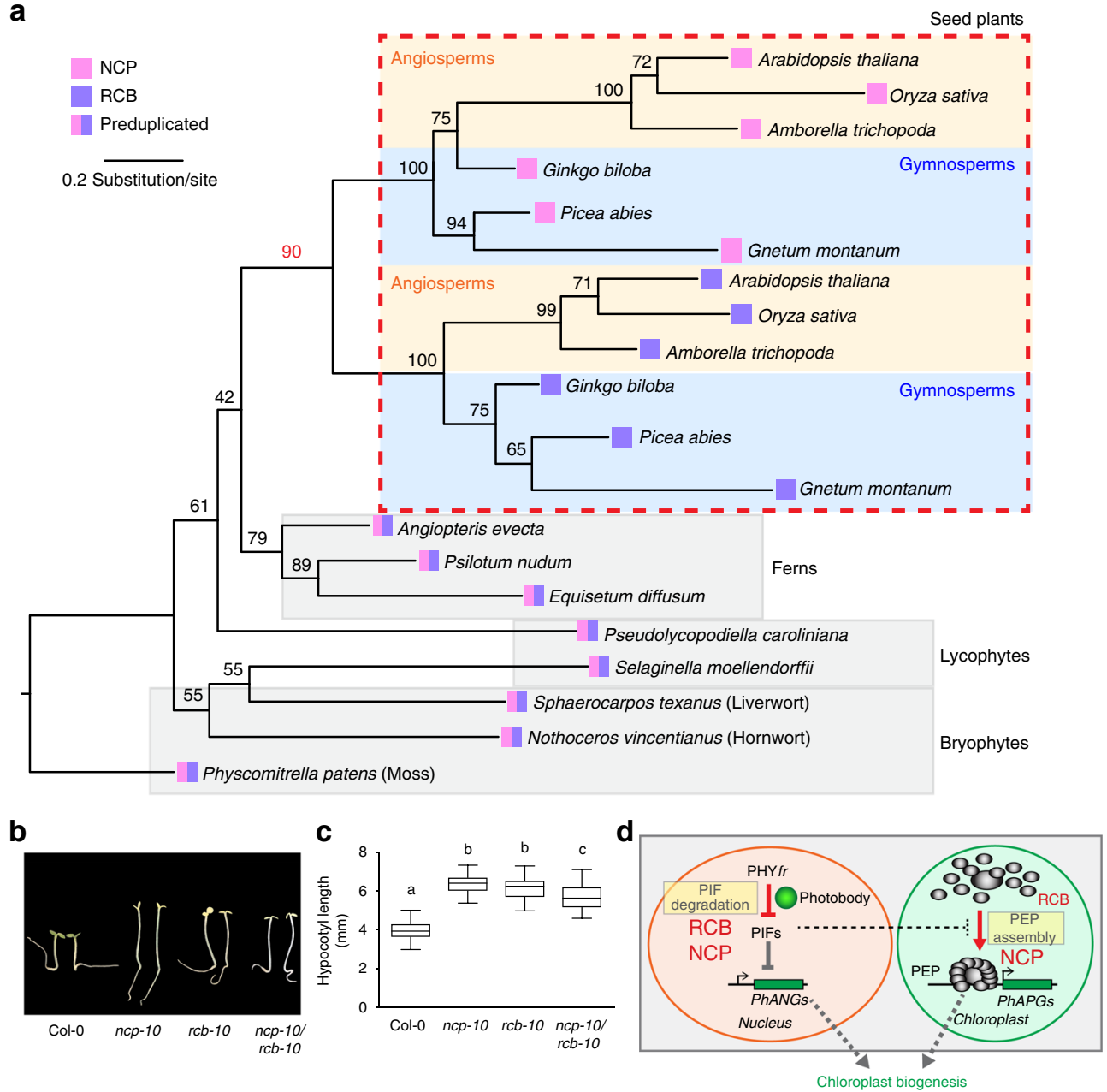

Fig. 5 NCP and RCB diverged in seed plants to adopt distinct roles in PHY signaling. a Maximum likelihood phylogenetic tree of NCP and RCB from seed plants, ferns, lycophytes, and bryophytes. The gene IDs of the sequences used for the phylogenetic analysis are listed in Supplementary Table 4. The phylogenetic tree was constructed using the Randomized Axelerated Maximum Likelihood method. The numbers at each node indicate the bootstrap value (\%) from 1000 replications. The length of the branches represents the extent of divergence based on the scale at the bottom. $\mathbf{b}$ Representative images of 4-day-old Col-0, ncp-10, rcb-10, and ncp-10/rcb-10 seedlings grown in $10 \mu \mathrm{mol} \mathrm{m} \mathrm{m}^{-2} \mathrm{~s}^{-1} \mathrm{R}$ light. c Box-and-whisker plots showing hypocotyl length measurements of the seedlings in $\mathbf{b}$. The boxes represent from the 25 th to the 75 th percentiles, and the bars represent the median values. Samples with different letters exhibit statistically significant differences in hypocotyl length (ANOVA, Tukey's HSD, $p \leq 0.001, n>40$ ). d Model for the PHY-mediated nucleus-to-plastid light signaling pathway for PhAPG activation. PHYs activate PhAPGs through a linked nuclear and plastidial dual-switching mechanism in which PHY-mediated PIF degradation in the nucleus triggers the assembly and activation of the PEP in plastids for PhAPG transcription. PIF degradation is the nodal signaling step governing the activation of PhAPGs and photosynthesis-associated nuclear-encoded genes (PhANGs). While RCB participates primarily in the nuclear switching mechanism, NCP controls both the nuclear and plastidial switches. The source data underlying the hypocotyl measurements in $\mathbf{c}$ are provided in the Source Data file

are removed, rescues $r c b-10$ 's defects in PEP assembly, PhAPG activation, and chloroplast biogenesis ${ }^{44}$. In contrast to RCB, NCP is required for controlling both the nuclear and plastidial switches for PhAPG activation. Although NCP had been reported previously for its essential role in chloroplast biogenesis ${ }^{35,36}$, the function of NCP in chloroplast biogenesis was still unknown. Different from the current view that NCP localizes only to plastids ${ }^{35}$, we show that NCP is dual-targeted to the plastids and the nucleus. The dual localization of NCP is supported by transiently expressed NCP-CFP-FLAG (Fig. 3a) and subcellular fractionation results using a NCP-HA-His/ncp-10 transgenic line (Fig. $3 b$ ). Although it is possible that the nuclear localization of $\mathrm{NCP}$ in these experiments could be due to overexpression of NCP, this is highly unlikely because a direct role of NCP in nuclear PHY signaling is also supported by the overwhelming genetic evidence. NCP is required for both PHYA and PHYB signaling (Fig. 2). RCB participates in the early light signaling events of photobody biogenesis (Fig. 3c, d). Moreover, both PIF1 and PIF3 accumulate in $n c p-10$ in the light (Fig. $3 \mathrm{e}$ ), and the long hypocotyl phenotype of $n c p-10$ was rescued in $n c p-10 / p$ ifq mutant (Fig. 4a), further supporting the notion that NCP is directly involved in PIF degradation in the nucleus. Intriguingly, the $n c p-$ 10/pifq mutant does not rescue the ncp-10's defects in PEP assembly, PhAPG activation, and chloroplast biogenesis (Fig. 4). Together, these results indicate that NCP facilitates both PHYmediated PIF degradation in the nucleus, possibly by promoting photobody biogenesis, as well as the assembly of the PEP in plastids (Fig. 5d).

We show that seed plants, including angiosperms and gymnosperms, contain both NCP and RCB (Fig. 5a). In non-seed land plants, including ferns and mosses, there is only one copy of NCP-like gene. It is intriguing that seed plants have evolved both 
a

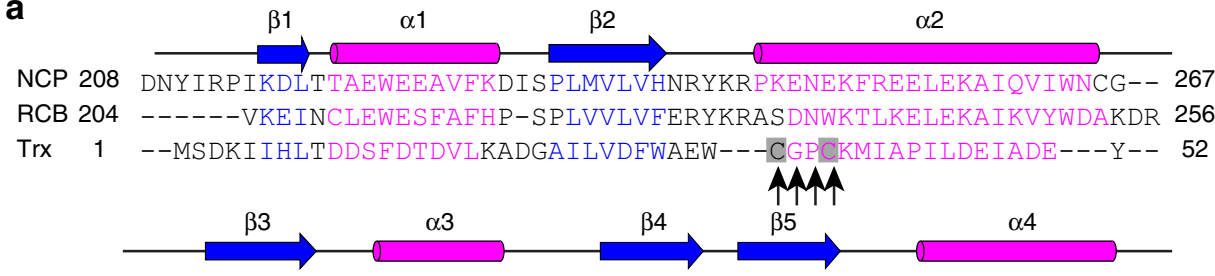

NCP 268 LPSPRCVAVDAVVETDLVSALKVSVFPEIIFTKAGKILYREKGIRTADELSKIMAFFYYG 326 RCB 257 LP-PRAVKIDLNIETDLAYALKAKECPQILFLRGNRILYREKDFRTADELVHMIAHFYYK 315 Trx 53 QGKLTVAKLNIDQNPGTAPKYGIRGIPTLLLFKNGEVAATKVGALSKGQLKEFLDANLA- 109

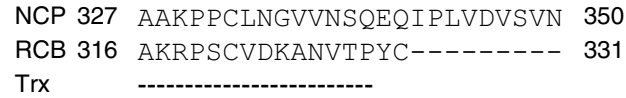

NCP 327 AAKPPCLNGVVNSQEQIPLVDVSVN 350 RCB 316 AKRPSCVDKANVTPYC--------- 331 Trx

b

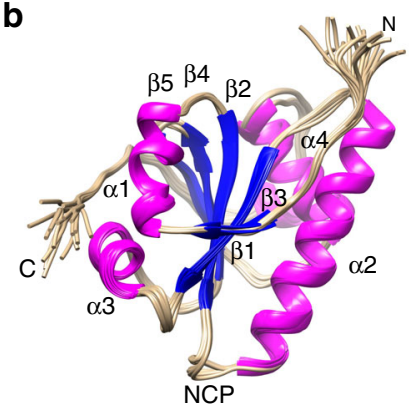

C

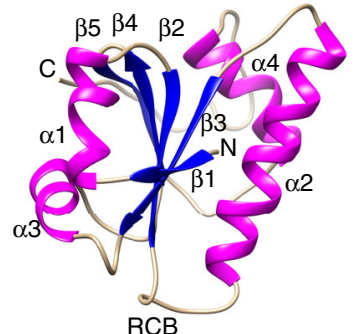

d

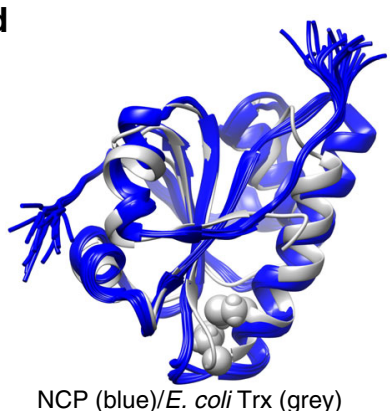

e

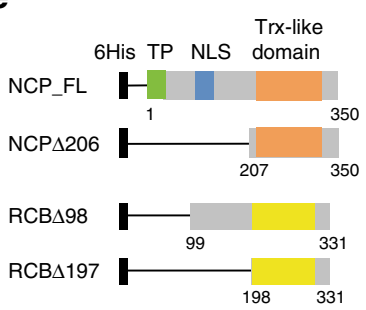

$\mathbf{f}$

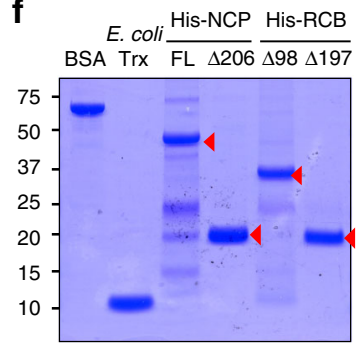

g

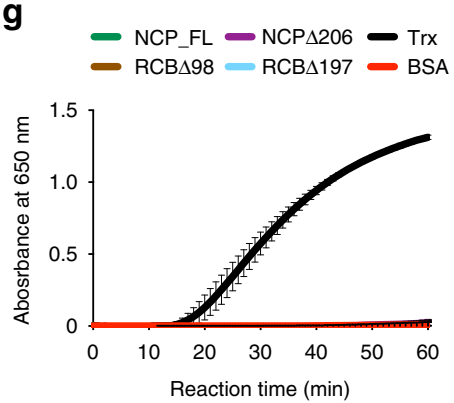

Fig. $6 \mathrm{NCP}$ and RCB structurally resemble E. coli Trx but lack reductase activity. a Sequence alignment of the Trx-like domains of NCP and RCB with that of E. coli Trx. Magenta characters represent alpha helices and blue characters represent beta sheets. The black arrows indicate the conserved catalytic -CysX-X-Cys- motif in E. coli Trx. b NMR structures of NCP's Trx-like domain (PDB ID: 6NE8). c Simulated structure of RCB's Trx-like domain based on the NMR structure of NCP. d Overlay of the structure of NCP's Trx-like domain with the crystal structure of E. coli Trx (PDB ID: 1XOB). The two cysteines in E. coli Trx are shown. e Schematic illustration of NCP and RCB fragments used for the in vitro Trx reductase assays. $f$ SDS-PAGE gels showing BSA (negative control), E. coli Trx (positive control), and purified His-tagged recombinant NCP and RCB fragments. $\mathbf{g}$ Trx activity assays showing that recombinant NCP and RCB proteins lack Trx activity. Trx activity was determined based on the increase in turbidity after the reduction of insulin, as measured by the absorbance at $650 \mathrm{~nm}^{53}$. E. coli Trx and BSA proteins were used as a positive control and a negative control, respectively. The source data of the Trx activity assay in $\mathbf{g}$ are provided in the Source Data file

$\mathrm{NCP}$ and RCB, which play non-redundant roles in PHY signaling (Fig. 5b, c). One possibility is that having both NCP and RCB can provide an additional regulatory mechanism for the lightdependent chloroplast biogenesis or greening in seed plants ${ }^{3,4}$. NCP and RCB might work in concert in the nucleus to promote PHYs to localize to photobodies for PIF degradation; for example, they could form a heteromeric protein complex. We will test this hypothesis in future investigations. The functions of NCP and RCB in plastids are likely distinct. Only NCP plays an essential role in PEP assembly and PhAPG activation in plastids (Fig. 4$)^{44}$. NCP and RCB show different localization patterns in plastids: while RCB is localized to the nucleoid, NCP is localized to the stroma (Fig. 3a) ${ }^{35}$. It is unclear how the localization patterns of these proteins relate to their platidial functions. Our previous work has identified another dual-targeted nuclear and plastidial protein, HMR, which also participates in the PHY-mediated control of PhAPG activation $19,28,41$. While nuclear HMR acts as a transcriptional activator interacting directly with PIFs to mediate PIF1 and PIF3 degradation, plastidial HMR, also called pTAC12, is an essential component of the PEP complex ${ }^{28,54,55}$. Our genetic studies have so far identified three dual-targeted nuclear/plastidial molecules in PHY signaling- $\mathrm{HMR}^{19,41}, \mathrm{RCB}^{44}$, and NCP (this study). One pressing upcoming task is to determine the regulation and mechanism of their dual localization, as well as to understand the significance of their dual-localization in PHY signaling and in nucleus-plastid communication.

NCP and RCB each contain a Trx-like domain. Although a previous study suggested that RCB's Trx domain possesses Trx reductase activity ${ }^{51}$, our results demonstrate convincingly that the Trx-like domains of NCP and RCB are structurally similar to that of E. coli Trx but lack reductase activity (Fig. 6a-c). Neither NCP nor RCB contains the universally conserved -Cys-X-X-Cys- 
Table 1 Structural statistics for the Trx-like domain of NCP

\section{NCP Trx-like domain (D208-N350)}

\begin{tabular}{|c|c|}
\hline NOE distance restraints & 6313 \\
\hline Short-range $(|\mathrm{i}-\mathrm{j}| \leq 1)$ & 2118 \\
\hline Medium-range $(1<|\mathrm{i}-\mathrm{j}| \leq 4)$ & 1457 \\
\hline Long-range $(|i-j| \geq 5)$ & 2738 \\
\hline Dihedral angle constraints ${ }^{c}$ & 245 \\
\hline Target function value & $2.34 \pm 0.05$ \\
\hline \multicolumn{2}{|l|}{ Ramachandran plot ${ }^{d}$} \\
\hline Favored region (98\%) & $88 \%$ \\
\hline Allowed region (>99.8\%) & $12 \%$ \\
\hline \multicolumn{2}{|l|}{ Mean pairwise RMSD (RCBL Y210-V347) } \\
\hline Backbone & $0.29 \pm 0.03 \AA$ \\
\hline Heavy atoms & $0.65 \pm 0.03 \AA$ \\
\hline $\begin{array}{l}\text { a None of these structures exhibit distance violations gre } \\
\text { violations greater than } 4^{\circ} \\
\text { bTwo constraints }\left(d_{H N-O} \leq 2.5 \AA \text { and } d_{N-O} \leq 3.5 \AA \text { ) are us }\right. \\
\text { "Dihedral angle constraints were generated by TALOS }+ \text { b } \\
\text { shifts }{ }^{76} \text {, and by analysis of NOE patterns } \\
d_{\text {MOLPROBITY was used to assess the quality of the struct }}\end{array}$ & $\begin{array}{l}\text { than } 0.4 \AA \text { or dihedral angle } \\
\text { or each identified hydrogen bor } \\
\text { on backbone atom chemical }\end{array}$ \\
\hline
\end{tabular}

catalytic motif required for reductase activity (Fig. $6 \mathrm{~d})^{50}$. Trx could participate in a regulatory mechanism independent of redox activity, depending on the ability to interact with other proteins to form functional protein complexes. For example, $E$. coli Trx that lacks the catalytic cysteine residues in its active site can enhance the processivity of the bacteriophage T7 DNA polymerase ${ }^{56,57}$; the active site in this case mediates the interaction with the T7 DNA polymerase ${ }^{58}$. Similarly, RCB and NCP could use their non-catalytic Trx-like domains for protein-protein interactions. Future investigations will test this hypothesis to determine the biochemical functions of NCP and RCB in nuclear PHY signaling as well as PEP assembly and activation.

\section{Methods}

Plant materials and growth conditions. The $P B G$ line in the Landsberg erecta (Ler) background has been previously reported ${ }^{31}$. The $n c p-1 / P B G$ mutant line was isolated from the tall-and-albino mutant screen and backcrossed to $P B G$ three times. The ncp-10 mutant (Col-0 background) was identified from GABI-Kat TDNA insertion line GK-518H0259 and obtained from the Arabidopsis Biological Resource Center (CS449718). $n c p-1$ was genotyped using a dCAPS (derived Cleaved Amplified Polymorphic Sequences) marker using the following PCR primers: forward: agagaaggcgattcaagtcatat, reverse: ctggaagtaataatgacccag. NdeI digestion of the PCR product yields 129-bp and 20-bp fragments for Ler and one 149-bp fragment for ncp-1. The Arabidopsis mutants phyB-9, phyA-211, and pifq, all in the Col-0 background, were used for the physiological studies. YHB, a constitutively active phyB mutant in the Ler background has been previously reported ${ }^{38}$. Seeds were surface-sterilized and plated on half-strength Murashige and Skoog (MS) growth medium with Gamborg's vitamins (MSP0506, Caisson Laboratories, North Logan, UT) containing $0.5 \mathrm{mM}$ MES pH 5.7 and $0.8 \%$ agar $(\mathrm{w} / \mathrm{v})\left(\mathrm{A} 038\right.$, Caisson Laboratories, North Logan, UT) ${ }^{19}$. Seeds were stratified in the dark at $4^{\circ} \mathrm{C}$ for 5 days. Seedlings were grown at $21^{\circ} \mathrm{C}$ in an LED chamber (Percival Scientific, Perry, IA) under the indicated light conditions. Fluence rates of light were measured using an Apogee PS200 spectroradiometer (Apogee instruments Inc., Logan, UT) and SpectraWiz software (StellarNet, Tampa, FL).

Hypocotyl length measurement. For the measurement of hypocotyl length, 4-dold seedlings grown under different light conditions were scanned using an Epson Perfection V700 photo scanner, and hypocotyl lengths were measured using NIH ImageJ software (https://imagej.nih.gov/ij/). Box-and-whisker plots of hypocotyl measurements were generated using Prism 7 software (GraphPad, San Diego, CA). Images of representative seedlings were captured using a Leica MZ FLIII stereo microscope (Leica microsystems Inc., Buffalo Grove, IL) and processed using Adobe Photoshop CC (Adobe Systems, Mountain View, CA).

Mutant generation. $P B G$ seeds were hydrated in $45 \mathrm{ml}$ of $\mathrm{ddH}_{2} \mathrm{O}$ with $0.005 \%$ Tween- 20 for $4 \mathrm{~h}$ and washed with $\mathrm{ddH}_{2} \mathrm{O}$ twice. The washed seeds were soaked in $1 \mathrm{mM} \mathrm{N}$-ethyl-N-nitrosourea or ethyl methanesulfonate solution for $15 \mathrm{~h}$ with rotation. Then, the seeds were thoroughly washed in $\mathrm{ddH}_{2} \mathrm{O}$, plated on MS growth media, and stratified at $4{ }^{\circ} \mathrm{C}$ for 4 days. The $\mathrm{M} 1$ seedlings were transferred to soil, and the M2 seeds were collected from individual M1 plants. The M2 generation was screened under monochromatic $\mathrm{R}$ light.
Genetic Mapping via SHOREmap. $n c p-1 / P B G$ (Ler) was crossed to Col-0 to generate an F2 mapping population. Genomic DNA from pools of more than 800 F2 seedlings with a tall-and-albino phenotype was extracted as follows ${ }^{60}$. Seedlings were ground in liquid nitrogen and resuspended in nuclear extraction buffer containing $10 \mathrm{mM}$ Tris-HCl pH 9.5, $10 \mathrm{mM}$ EDTA pH 8.0, $100 \mathrm{mM} \mathrm{KCl}, 500 \mathrm{mM}$ sucrose, $4 \mathrm{mM}$ spermidine, $1 \mathrm{mM}$ spermine, and $0.1 \% \beta$-mercaptoethanol. Two mililiter of lysis buffer containing $10 \%$ Triton X-100 in nuclei extraction buffer was added into the homogenized tissues. After incubation on ice for $2 \mathrm{~min}$, the homogenate was centrifuged at $2000 \times g$ at $4{ }^{\circ} \mathrm{C}$ for $10 \mathrm{~min}$. The nuclei pellet was

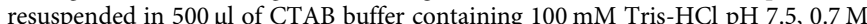
$\mathrm{NaCl}, 10 \mathrm{mM}$ EDTA pH 8.0, 1\% CTAB, and $1 \% \beta$-mercaptoethanol. After incubation at $60^{\circ} \mathrm{C}$ for $30 \mathrm{~min}$, genomic DNA was extracted with chloroform/isoamyl alcohol (24:1) and precipitated with isopropanol by centrifugation at $20,000 \times g$ at $4^{\circ} \mathrm{C}$ for $10 \mathrm{~min}$. Illumina paired-end libraries with 300 -bp insert sizes were constructed per the manufacturer's instructions. Eighty-base paired-end reads were generated on an Illumina Genome Analyzer II, targeting approximately $25 \times$ genome coverage. The polymorphisms, including SNPs, indels up to $3 \mathrm{bp}$, and large deletions, were identified using SHOREmap ${ }^{61}$. Genomic regions enriched for mutant parental markers were identified with SHOREmap 62,63 . Variants in the final mapping interval that were absent from the Ler background and that were predicted to have a large impact on ORF integrity were prioritized as candidate mutations.

Plasmid construction and generation of transgenic plants. All the primers used for plasmid construction are listed in Supplementary Table 1. The $p C H F 1$ $U B Q 10 p:: N C P-H A-H i s$ construct was generated by cloning the UBQ10 promoter and the full-length coding sequence of NCP into the EcoRI and PstI sites of $p C H F 1-$ HA-His vector using Gibson assembly (New England Biolabs, Ipswich, MA); the construct was prepared by inserting a DNA fragment encoding (PT)4P-3HA-6His into the PstI and SalI sites of the $p C H F 1$ vector ${ }^{64}$. Transgenic lines were generated by transforming $n c p-10$ heterozygous plants with Agrobacterium tumefaciens strain GV3101 containing the $p C H F 1-U B Q 10:: N C P-H A-H i s$ construct. The T1 transgenic plants were selected on half-strength MS medium containing $100 \mu \mathrm{g} / \mathrm{ml}$ gentamycin and screened for transgenic plants with a homozygous $n c p-10$ mutation. The T2 lines with a single-locus insertion status of the transgene were selected based on a 3:1 segregation ratio for gentamycin resistance. The T3 generation plants homozygous for the transgene were used for the experiments.

The NCP-CFP construct used for the tobacco transient expression assay was generated by amplifying the coding sequences of NCP and CFP-FLAG and inserting them into the XmaI and XbaI sites of the $p C H F 3$ vector. The NCP-CFP construct was transformed into Agrobacterium tumefaciens strain GV3101 for the transient expression assay. The constructs used for in vitro transcribed and translated HA-and His-tagged NCP or NCP $\triangle 48$ (deletion of N-terminal 48 aminoacid transit peptide) was generated by amplifying the NCP-HA-His fragments and inserting them into EcoRI and BamHI sites of the $p C M X-P L 2$ vector.

The constructs used for expressing N-terminally His-tagged NCP and RCB proteins in $E$. coli were made in the $p E T 15 b$ and $p E T 28 a$ vectors, respectively. NCP or NCP $\Delta 206$ (Trx-like domain only, aa 207-350) were amplified by PCR and ligated into the NdeI and XhoI sites of the $p E T 15 b$ vector using T4 DNA ligase. RCB $\triangle 98$ (aa 99-331) and RCB $\Delta 197$ (Trx-like domain only, aa 198-331) were amplified and inserted into the BamHI and HindIII sites of the pET28a vector using Gibson assembly.

RNA extraction and quantitative PCR. Total RNA from seedlings of the indicated genotypes and growth conditions was isolated using a Quick-RNA MiniPrep Kit with on-column DNase I treatment (Zymo Research, Irvine, CA). cDNA was synthesized using Superscript II First-strand cDNA Synthesis Kit (ThermoFisher Scientific, Waltham, MA). Oligo(dT) primers were used for the analysis of nuclear gene expression, and a mixture of oligo(dT) and plastidial-gene-specific primers was used for the analysis of plastidial genes. qRT-PCR was performed with FastStart Universal SYBR Green Master Mix on a LightCycler 96 System (Roche, Basel, Switzerland). The transcript level of each gene was normalized to that of PP2A. All primers used for qRT-PCR and cDNA synthesis are listed in Supplementary Tables 2 and 3

Phylogenetic analysis. We acquired the NCP-like sequences of seed plants (Arabidopsis thaliana, Oryza sativa, Amborella trichopoda, Ginkgo biloba, Picea abies, Gnetum montanum) from implemented BLASTp from Phytozome or individual genome portals ${ }^{65,66}$. We obtained the NCP-like sequences in ferns (Angiopteris evecta, Psilotum nudum, Equisetum diffusum), lycophytes (Pseudolycopodiella caroliniana, S. moellendorffi), and bryophytes [Nothoceros vincentianus (a hornwort), Sphaerocarpos Texanus (a liverwort), and Physcomitrella patens] from transcriptome data generated as part of the One Thousand Plants Project (www.onekp.com) ${ }^{67}$ using transcriptome mining based on the BlueDevil Python pipeline ${ }^{68}$. The homologs of NCP and RCB from 6 representative seed plants were BLASTed and obtained from Phytozome ${ }^{65}$, Congenie (congenie.org) ${ }^{66}$, and the Amborella Genome Database (www.amborella.org) ${ }^{69}$ (Supplementary Table 4). The homologous sequences from ferns, bryophytes, and lycophytes were mined using the Python pipeline BlueDevil ${ }^{68}$ from transcriptomes generated from One 
Thousand Plants Project ${ }^{67}$. The nucleotide sequences obtained from the transcriptomes were translated into amino acid sequences, including 20 sequences from 14 species. The sequences were aligned using MUSCLE ${ }^{70}$. To decrease ambiguities in sequence alignment, we only included the conserved Trx-like domain, and ambiguously aligned regions were manually removed before the phylogenetic analysis. The final alignment included 597 nucleotide sites. All alignments are available at Fig. Share (https://figshare.com/s/18f43720186936a3effd). The processed polypeptide sequences of NCP and RCB paralogues were used for phylogenetic tree construction. The best substitution model and partition scheme were inferred using PartitionFinder v1.1.0 $0^{71}$. The best maximum-likelihood tree was generated using RAxML version $7.2 .8^{47}$ with substitution model GTRGAMMAI, and multiparametric bootstrapping was conducted using RAxML with 1000 replicates. The phylogenetic tree generated was visualized in FigTree v.1.4.2, and Adobe Photoshop CC software (Adobe Systems, Inc., San Jose, CA) was used to label the name of each species.

Transient expression in $\boldsymbol{N}$. benthamiana. Fluorescent-protein-tagged NCP was transiently expressed in $N$. benthamiana leaves. The $p C H F 3-N C P-C F P-F L A G$ plasmid was transformed by electroporation into Agrobacterium tumefaciens strain GV3101. Agrobacterium cells were grown overnight, pelleted, and resuspended in a volume of infiltration buffer equal to half the volume of the original culture. The infiltration buffer contained $10 \mathrm{mM} \mathrm{MgCl} 2,10 \mathrm{mM}$ MES pH 5.7, and $200 \mu \mathrm{M}$ acetosyringone ( $4^{\prime}$-hydroxy-3', $5^{\prime}$-dimethosyacetophenone). Cells were diluted to the O.D.600 of 1.0. Cells were infiltrated into the abaxial side of Nicotiana benthamiana leaves. Samples were collected at $72 \mathrm{~h}$ after infiltration and stained with DAPI for microscopic analyses.

\section{Confocal imaging and quantification of photobody morphology. For the} quantification of photobody morphology, seedlings were mounted on Superfrost slides (VWR, Radnor, PA) using $\mathrm{ddH}_{2} \mathrm{O}$ and $22 \times 40 \mathrm{~mm}$ coverslips (no. 1.5, VWR, Radnor, PA). The nuclei from the epidermal cells of the top third of the hypocotyl were imaged using a Zeiss LSM 510 inverted confocal microscope (Carl Zeiss, Thornwood, NY). GFP signal was detected using a 100× Plan-Apochromat oil immersion objective, $488 \mathrm{~nm}$ excitation from an argon laser, and a 505-550 nm bandpass filter. Images were collected using LSM 510 software version 4.2. Images were processed using Adobe Photoshop CC software (Adobe Systems, Inc., San Jose, CA). To determine the size and number of photobodies, the volume of photobodies was calculated using the object analyzer tool in Huygens Essentials (Scientific Volume Imaging, The Netherlands). For each nucleus, the information on the photobodies and box-and-whisker plots was sorted and calculated using Graphpad Prism 7. NCP-CFP-FLAG was detected using $458 \mathrm{~nm}$ excitation from an argon laserand a $470-500 \mathrm{~nm}$ bandpass filter.

Nuclear and chloroplast fractionation. For chloroplast fractionation, 2-d-old NCP-HA-His seedlings grown in Rc were frozen and homogenized in liquid nitrogen. One gram of seedlings was extracted in $2 \mathrm{ml}$ of cold grinding buffer (GB, $50 \mathrm{mM}$ HEPES-KOH pH 7.3, 0.33 M sorbitol, $0.1 \%$ BSA, 1 mM MnCl $2,2 \mathrm{mM}$ EDTA, and $1 \times$ protease inhibitor cocktail (Millipore Sigma, St. Louis, MO) ${ }^{19}$. The plant extract was filtered through two layers of Miracloth (Millipore Sigma, St. Louis, MO) and centrifuged for $2 \mathrm{~min}$ at $2600 \times g$ to spin down the chloroplasts. The crude chloroplasts were resuspended in $0.2 \mathrm{~mL}$ of GB buffer and fractionated on a Percoll (Millipore Sigma, St. Louis, MO) step gradient ( 80 and $40 \%$ ) by centrifugation for $10 \mathrm{~min}$ at $2600 \times \mathrm{g}$. Intact chloroplasts were obtained from the interface between the 80 and $40 \%$ Percoll.

For nuclear fractionation, seedlings were frozen in liquid nitrogen and homogenized with nuclei extraction buffer containing $20 \mathrm{mM}$ PIPES-KOH pH 7.0, $10 \mathrm{mM} \mathrm{MgCl}, 12 \%$ hexylene glycol, $0.25 \%$ Triton X-100, $5 \mathrm{mM} \beta$ mercaptoethanol, and $1 \times$ protease inhibitor cocktail. The lysate was filtered through two layers of Miracloth. The filtered lysate was loaded on top of $2 \mathrm{ml}$ of $30 \%$ Percoll in $5 \mathrm{mM}$ PIPES-KOH pH 7.0, $10 \mathrm{mM} \mathrm{MgCl}_{2}, 3 \%$ hexylene glycol, $0.25 \%$ Triton X-100, and $5 \mathrm{mM} \beta$-mercaptoethanol, and centrifuged at $700 \times g$ for $5 \mathrm{~min}$ at $4^{\circ} \mathrm{C}$. The fractionated nuclear pellet was dissolved in nuclei extraction buffer. Protein extracts from the chloroplast and nuclear fractions were resolved via SDS-PAGE and analyzed by immunoblot.

Protein extraction and immunoblot analysis. For PIF1 and PIF3 protein extraction, seedlings were ground directly in extraction buffer in a 1:3 $(\mathrm{mg} / \mu \mathrm{l})$ ratio, boiled for $10 \mathrm{~min}$ and then centrifuged at $15,000 \times \mathrm{g}$ for $10 \mathrm{~min}$ at room temperature ${ }^{55}$. The extraction buffer consisted of $100 \mathrm{mM}$ Tris-HCl, pH 7.5, $100 \mathrm{mM}$ $\mathrm{NaCl}, 5 \mathrm{mM}$ EDTA, pH 8.0, 5\% SDS, 20\% glycerol, $20 \mathrm{mM}$ dithiothreitol (DTT), 40 $\mathrm{mM} \beta$-mercaptoethanol, $2 \mathrm{mM}$ PMSF, $1 \times$ protease inhibitor cocktail, $80 \mu \mathrm{M}$ MG132 (Millipore Sigma, St. Louis, MO), 80 MM MG115 (Millipore Sigma, St. Louis, MO), $1 \%$ phosphatase inhibitor cocktail 3 (Millipore Sigma, St. Louis, MO), and $10 \mathrm{mM}$ N-ethylmaleimide. Protein extracts were separated on an SDS-PAGE mini-gel and transferred onto a polyvinylidene difluoride (PVDF) membrane. The membrane was blocked in $2 \%$ non-fat milk in $1 \times$ TBS, probed with the indicated primary antibodies, and then incubated with anti-rabbit or anti-mouse secondary antibodies conjugated with horseradish peroxidase. For the fractionation experiments, in vitro translated NCP-HA-His proteins were produced using the TNT T7-
Coupled Reticulocyte Lysate System (Promega) according to the manufacturer's protocol and detected using mouse anti-HA antibodies (11583816001, Millipore Sigma, St. Louis, MO). The purity of the chloroplast and nuclear fractions was monitored using antibodies against chloroplast ferredoxin:sulfite reductase $(\mathrm{SiR})^{72}$ and mouse monoclonal anti-RNA polymerase II (Pol II) antibodies (8WG16, Biolegend, San Diego, CA), respectively. Both anti-SiR and anti-Pol II antibodies were used at a 1:1000 dilution. Rabbit anti-PIF $1^{19}$ and anti-PIF3 ${ }^{19}$ polyclonal antibodies were used at a 1:500 dilution ${ }^{19}$. Anti-RPN6 antibodies (BMLPW8370-0100, Enzo Life Sciences, Farmingdale, NY) were used at a 1:1000 dilution. For blue native gels, mouse monoclonal anti-rpoB (PHY1700, PhytoAB Inc. Redwood City, CA) and rabbit polyclonal anti-HMR ${ }^{19}$ antibodies were used at a 1:1000 dilution to detect the PEP complex. Secondary antibodies including antimouse (1706516, Bio-Rad, Hercules, CA) and anti-rabbit (1706515, Bio-Rad, Hercules, CA) were used at a 1:5000 dilution. The signals were detected with a chemiluminescence reaction using SuperSignal West Dura Extended Duration Chemiluminescent Substrate (ThermoFisher Scientific, Waltham, MA).

Protein purification and NMR spectroscopy. The DNA fragment encoding the Trx-like domain of NCP (residues 208-350) was PCR-amplified and cloned into a modified $p E T 15 b$ vector to yield an $\mathrm{N}$-terminally $\mathrm{His}_{10}$-tagged protein with a TEV site between the $\mathrm{His}_{10}$-tag and the thioredoxin-like domain of NCP. The His ${ }_{10^{-}}$ tagged NCP was overexpressed in the BL21 (DE3) STAR E. coli strain (ThermoFisher Scientific, Waltham, MA). The cultures were grown at $37^{\circ} \mathrm{C}$ until the O.D. 600 reached 0.6-0.8. The cells were induced with $0.6 \mathrm{mM}$ IPTG at $20^{\circ} \mathrm{C}$ for $20 \mathrm{~h}$. The harvested cells were purified via $\mathrm{Ni}^{2+}$-NTA affinity chromatography. After TEV digestion to remove the $\mathrm{His}_{10}$-tag, NCP was further purified by size-exclusion chromatography (Superdex 75, GE Healthcare Life Sciences, Marlborough, MA). Isotopically enriched proteins were overexpressed in $\mathrm{M} 9$ media using ${ }^{15} \mathrm{~N}-\mathrm{NH}_{4} \mathrm{Cl}$ and ${ }^{13} \mathrm{C}$-glucose as the sole nitrogen and carbon sources (Cambridge Isotope Laboratories, Tewksbury, MA). The final NMR samples contained $\sim 1.2 \mathrm{mM}$ protein in a buffer containing $25 \mathrm{mM}$ HEPES, $50 \mathrm{mM} \mathrm{KCl} \mathrm{pH} \mathrm{7.0,} \mathrm{and} 10 \mathrm{mM}$ DTT in either $90 \% \mathrm{H}_{2} \mathrm{O} / 10 \% \mathrm{D}_{2} \mathrm{O}$ or in $100 \% \mathrm{D}_{2} \mathrm{O}$.

NMR experiments were conducted using Agilent INOVA 600 or $800 \mathrm{MHz}$ spectrometers at $35^{\circ} \mathrm{C}^{73}$. Backbone and side-chain resonances were assigned based on standard three-dimensional triple-resonance experiments and sparsely sampled highresolution 4D HCCH-TOCSY and HCCONH TOCSY experiments. Distance constraints were derived from 3D ${ }^{15} \mathrm{~N}-\mathrm{NOESY}$, sparsely sampled $4 \mathrm{D}{ }^{13} \mathrm{C}-\mathrm{HMQC}-$ NOESY- ${ }^{15} \mathrm{~N}-\mathrm{HSQC}$ and $4 \mathrm{D}{ }^{13} \mathrm{C}$-HMQC-NOESY ${ }^{13} \mathrm{C}-\mathrm{HSQC}$ experiments. The NMR data were processed with NMRPipe and analyzed with SPARKY ${ }^{74,75}$. TALOS+ analysis was used to derive the dihedral angle restraints, and CYANA was used to calculate the structures ${ }^{76,77}$. The final NCP ensemble of 20 structures displayed no NOE violations $>0.3 \AA$ and no dihedral angle violations $>3^{\circ}$. The quality of the NMR ensemble was evaluated by MolProbity ${ }^{78}$.

Thioredoxin activity assay. Trx activity assays were carried out in reaction buffer containing $0.1 \mathrm{M}$ potassium phosphate $\mathrm{pH} 7.0,2 \mathrm{mM}$ EDTA, $0.5 \mathrm{mM}$ DTT, 0.167 $\mathrm{mM}$ insulin as the substrate, and $1 \mu \mathrm{M}$ His-tagged NCP or His-tagged RCB protein fragments ${ }^{53}$. E. coli Trx (Millipore Sigma, St. Louis, MO) and BSA (Millipore Sigma, St. Louis, MO) were used as positive and negative controls, respectively. The reaction mixtures were incubated with DTT for $5 \mathrm{~min}$, and the reaction was started by adding insulin. The reduction of insulin generated turbidity, which was detected by measuring the absorbance at $650 \mathrm{~nm}$ every $\min$ for $1 \mathrm{~h}$ using a DU730 Life Science UV/Vis Spectrophotometer (Beckman Coulter, Inc., Brea, CA).

Blue native gel electrophoresis. Seedlings grown under the indicated conditions were ground in liquid nitrogen and resuspended in 3 volumes of BN-Lysis buffer (100 mM Tris-Cl, pH7.2; 10 mM MgCl2; 25\% glycerol; $1 \%$ Triton X-100; $10 \mathrm{M}$ $\mathrm{NaF} ; 5 \mathrm{mM} \beta$-mercaptoethanol; $1 \times$ protease inhibitor cocktail) ${ }^{79}$. The protein extracts were divided to two tubes: one for blue-native PAGE and the other for SDS-PAGE. For blue-native PAGE, the protein extracts were mixed with BNSample buffer ( $1 \times$ NativePAGE Sample Buffer, $50 \mathrm{mM}$ 6-aminocaproic acid, $1 \% n$ dodecyl $\beta$-D-maltoside, and benzonase) and incubated for $60 \mathrm{~min}$ at room temperature to degrade DNA/RNA and further solubilize the PEP complex. Samples were mixed with $0.25 \%$ NativePAGE Coomassie blue G-250 Sample Additive and centrifuged at $17,500 \times g$ for $10 \mathrm{~min}$ at $4^{\circ} \mathrm{C}$. Proteins from the supernatant were separated on a $4-16 \%$ NativePAGE Bis-Tris Protein Gel (ThermoFisher Scientific, Waltham, MA) according to the manufacturer's protocol (ThermoFisher Scientific, Waltham, MA) and with the following modifications. NativeMark Unstained Protein Standard (ThermoFisher Scientific, Waltham, MA) was used to determine protein size in blue-native PAGE. Briefly, electrophoresis was performed at a constant $30-40 \mathrm{~V}$ for $3 \mathrm{~h}$ at $4{ }^{\circ} \mathrm{C}$ until the blue dye migrated through one third of the gel. The Dark Blue Cathode Buffer was replaced with Light Blue Cathode Buffer, and electrophoresis continued at a constant $20-25 \mathrm{~V}$ overnight (16-18 h) at $4{ }^{\circ} \mathrm{C}$. After electrophoresis was complete, the separated proteins were transferred onto a PVDF membrane using $1 \times$ NuPAGE Transfer Buffer (ThermoFisher Scientific, Waltham, MA) at a constant $70 \mathrm{~V}$ for $7 \mathrm{~h}$ at $4{ }^{\circ} \mathrm{C}$. After transfer, the membrane was fixed with fixation buffer (25\% methanol, $10 \%$ acetic acid) for $15 \mathrm{~min}$ and washed with water. The membrane was incubated with methanol for 3 min to destain the dye, and then immunoblotting proceeded. To determine total 
amount of rpoB and HMR proteins, samples were mixed with $1 \times$ SDS Laemmli buffer containing $10 \%$ SDS and $50 \mathrm{mM}$ 6-aminocaproic acid, $100 \mathrm{mM}$ DTT, and $20 \mathrm{mM}$ beta-mercaptoethanol, immediately boiled for $10 \mathrm{~min}$, and then centrifuged at $17,500 \times g$ for $10 \mathrm{~min}$ at room temperature. Proteins from the supernatant were separated via SDS-PAGE and analyzed by immunoblot.

Reporting summary. Further information on research design is available in the Nature Research Reporting Summary linked to this article.

\section{Data availability}

Arabidopsis mutants and transgenic lines, as well as plasmids generated during the current study, are available from the corresponding author upon reasonable request. The NMR assignments and the coordinate of the Trx-like domain of NCP has been deposited to Biological Magnetic Resonance Bank (30551) and RCSB Protein Data Bank (PDB ID: $6 \mathrm{NE} 8$ ), respectively. The source data underlying Figs. 1b, 1e, 1f, 2b, 2d, 2f, 2h, 2j, 3b, 3d, $3 e, 4 b-d, 5 c$, and $6 \mathrm{~g}$ and Supplementary Figs. $1 \mathrm{~b}, 1 \mathrm{c}, 2 \mathrm{~b}, 2 \mathrm{~d}, 3 \mathrm{~b}$, and $3 \mathrm{c}$ are provided as a Source Data file.

Received: 15 January 2019 Accepted: 17 May 2019

Published online: 14 June 2019

\section{References}

1. Reyes-Prieto, A., Weber, A. P. M. \& Bhattacharya, D. The origin and establishment of the plastid in algae and plants. Annu. Rev. Genet. 41, 147-168 (2007).

2. Bock, R. Structure, function, and inheritance of plastid genomes. In Cell and Molecular Biology of Plastids (ed Bock, R.) 29-63 (Springer, Berlin Heidelberg, 2007).

3. Kirk, J. T. O. \& Tilney-Bassett, R. A. E. The plastids: their chemistry, structure, growth and inheritance. (Elsevier, North-Holland, 1978).

4. Armstrong, G. A. Greening in the dark: light-independent chlorophyll biosynthesis from anoxygenic photosynthetic bacteria to gymnosperms. J. Photochem. Photobiol. B 43, 87-100 (1998).

5. Chen, M., Chory, J. \& Fankhauser, C. Light signal transduction in higher plants. Annu. Rev. Genet. 38, 87-117 (2004).

6. Strasser, B., Sanchez-Lamas, M., Yanovsky, M. J., Casal, J. J. \& Cerdan, P. D. Arabidopsis thaliana life without phytochromes. Proc. Natl Acad. Sci. USA 107, 4776-4781 (2010).

7. $\mathrm{Hu}, \mathrm{W}$. et al. Unanticipated regulatory roles for Arabidopsis phytochromes revealed by null mutant analysis. Proc. Natl Acad. Sci. USA 110, 1542-1547 (2013).

8. Takano, M. et al. Phytochromes are the sole photoreceptors for perceiving red/ far-red light in rice. Proc. Natl Acad. Sci. USA 106, 14705-14710 (2009).

9. Rockwell, N. C., Su, Y. S. \& Lagarias, J. C. Phytochrome structure and signaling mechanisms. Annu. Rev. Plant Biol. 57, 837-858 (2006).

10. Sharrock, R. A. \& Quail, P. H. Novel phytochrome sequences in Arabidopsis thaliana: structure, evolution, and differential expression of a plant regulatory photoreceptor family. Genes Dev. 3, 1745-1757 (1989).

11. Van Buskirk, E. K., Decker, P. V. \& Chen, M. Photobodies in light signaling. Plant Physiol. 158, 52-60 (2012).

12. Bauer, D. et al. CONSTITUTIVE PHOTOMORPHOGENESIS 1 and multiple photoreceptors control degradation of PHYTOCHROME INTERACTING FACTOR 3, a transcription factor required for light signaling in Arabidopsis. Plant Cell 16, 1433-1445 (2004).

13. Al-Sady, B., Ni, W., Kircher, S., Schafer, E. \& Quail, P. H. Photoactivated phytochrome induces rapid PIF3 phosphorylation prior to proteasomemediated degradation. Mol. Cell 23, 439-446 (2006).

14. Leivar, P. \& Quail, P. H. PIFs: pivotal components in a cellular signaling hub. Trends Plant Sci. 16, 19-28 (2011).

15. Leivar, P. et al. Definition of early transcriptional circuitry involved in lightinduced reversal of PIF-imposed repression of photomorphogenesis in young Arabidopsis seedlings. Plant Cell 21, 3535-3553 (2009).

16. Shin, J. et al. Phytochromes promote seedling light responses by inhibiting four negatively-acting phytochrome-interacting factors. Proc. Natl Acad. Sci. USA 106, 7660-7665 (2009).

17. Qiu, Y. et al. Mechanism of early light signaling by the carboxy-terminal output module of Arabidopsis phytochrome B. Nat. Commun. 8, 1905 (2017).

18. Park, E., Kim, Y. \& Choi, G. Phytochrome B requires PIF degradation and sequestration to induce light responses across a wide range of light conditions. Plant Cell 30, 1277-1292 (2018).

19. Chen, M. et al. Arabidopsis HEMERA/pTAC12 initiates photomorphogenesis by phytochromes. Cell 141, 1230-1240 (2010).

20. Van Buskirk, E. K., Reddy, A. K., Nagatani, A. \& Chen, M. Photobody localization of phytochrome B is tightly correlated with prolonged and light- dependent inhibition of hypocotyl elongation in the dark. Plant Physiol. $\mathbf{1 6 5}$ 595-607 (2014).

21. Klein, R. R. \& Mullet, J. E. Light-induced transcription of chloroplast genes. psbA transcription is differentially enhanced in illuminated barley. J. Biol. Chem. 265, 1895-1902 (1990).

22. Deng, X. W. \& Gruissem, W. Control of plastid gene expression during development: the limited role of transcriptional regulation. Cell 49, 379-387 (1987).

23. Liere, K., Weihe, A. \& Borner, T. The transcription machineries of plant mitochondria and chloroplasts: Composition, function, and regulation. $J$. Plant Physiol. 168, 1345-1360 (2011).

24. Allison, L. A., Simon, L. D. \& Maliga, P. Deletion of rpoB reveals a second distinct transcription system in plastids of higher plants. EMBO J. 15, 2802-2809 (1996)

25. Hajdukiewicz, P. T., Allison, L. A. \& Maliga, P. The two RNA polymerases encoded by the nuclear and the plastid compartments transcribe distinct groups of genes in tobacco plastids. EMBO J. 16, 4041-4048 (1997).

26. Taylor, W. C. Regulatory interactions between nuclear and plastid genomes. Annu. Rev. Plant Physiol. Plant Mol. Biol. 40, 211-233 (1989).

27. Chen, M. \& Chory, J. Phytochrome signaling mechanisms and the control of plant development. Trends Cell Biol. 21, 664-671 (2011).

28. Pfalz, J., Liere, K., Kandlbinder, A., Dietz, K. J. \& Oelmuller, R. pTAC2, -6, and -12 are components of the transcriptionally active plastid chromosome that are required for plastid gene expression. Plant Cell 18, 176-197 (2006).

29. Borthwick, H. A., Hendricks, S. B. \& Parker, M. W. Action spectrum for inhibition of stem growth in dark-grown seedlings of albino and nonalbino barley (Hordeum vulgare). Bot. Gaz. 113, 95-105 (1951).

30. Jabben, M. \& Deitzer, G. F. Effects of the herbicide San 9789 on photomorphogenic responses. Plant Physiol. 63, 481-485 (1979).

31. Yamaguchi, R., Nakamura, M., Mochizuki, N., Kay, S. A. \& Nagatani, A. Light-dependent translocation of a phytochrome B-GFP fusion protein to the nucleus in transgenic Arabidopsis. J. Cell Biol. 145, 437-445 (1999).

32. Kelley, L. A., Mezulis, S., Yates, C. M., Wass, M. N. \& Sternberg, M. J. E. The Phyre2 web portal for protein modeling, prediction and analysis. Nat. Protoc. 10, 845-858 (2015).

33. Emanuelsson, O., Nielsen, H. \& Heijne, G. V. ChloroP, a neural networkbased method for predicting chloroplast transit peptides and their cleavage sites. Protein Sci. 8, 978-984 (1999).

34. Kosugi, S. et al. Six classes of nuclear localization signals specific to different binding grooves of importin a. J. Biol. Chem. 284, 478-485 (2009).

35. Qiao, J., Ma, C., Wimmelbacher, M., Bornke, F. \& Luo, M. Two novel proteins, MRL7 and its paralog MRL7-L, have essential but functionally distinct roles in chloroplast development and are involved in plastid gene expression regulation in Arabidopsis. Plant Cell Physiol. 52, 1017-1030 (2011).

36. Powikrowska, M. et al. SVR4 (suppressor of variegation 4) and SVR4-like: two proteins with a role in proper organization of the chloroplast genetic machinery. Physiol. Plant. 150, 477-492 (2014).

37. Nagy, F. \& Schafer, E. Phytochromes control photomorphogenesis by differentially regulated, interacting signaling pathways in higher plants. Annu. Rev. Plant Biol. 53, 329-355 (2002).

38. Su, Y.-S. \& Lagarias, J. C. Light-independent phytochrome signaling mediated by dominant GAF domain tyrosine mutants of Arabidopsis phytochromes in transgenic plants. Plant Cell 19, 2124-2139 (2007).

39. Huq, E., Al-Sady, B. \& Quail, P. H. Nuclear translocation of the photoreceptor phytochrome B is necessary for its biological function in seedling photomorphogenesis. Plant J. 35, 660-664 (2003).

40. Hiltbrunner, A. et al. Nuclear accumulation of the phytochrome A photoreceptor requires FHY1. Curr. Biol. 15, 2125-2130 (2005).

41. Nevarez, P. A. et al. Mechanism of dual targeting of the phytochrome signaling component HEMERA/pTAC12 to plastids and the nucleus. Plant Physiol. 173, 1953-1966 (2017).

42. Chen, M., Schwab, R. \& Chory, J. Characterization of the requirements for localization of phytochrome B to nuclear bodies. Proc. Natl Acad. Sci. USA 100, 14493-14498 (2003)

43. Steiner, S., Schröter, Y., Pfalz, J. \& Pfannschmidt, T. Identification of essential subunits in the plastid-encoded RNA polymerase complex reveals building blocks for proper plastid development. Plant Physiol. 157, 1043-1055 (2011).

44. Yoo, C. Y. et al. Phytochrome activates the plastid-encoded RNA polymerase for chloroplast biogenesis via nucleus-to-plastid signaling. Nat. Commun. 10, https://doi.org/10.1038/s41467-019-10518-0. (2019).

45. Martín, G. et al. Phytochrome and retrograde signaling pathways converge to antagonistically regulate a light-induced transcriptional network. Nat. Commun. 7, 11431 (2016).

46. Yu, F. et al. SUPPRESSOR OF VARIEGATION4, a new var2 suppressor locus encodes a pioneer protein that is required for chloroplast biogenesis. $\mathrm{Mol}$. Plant 4, 229-240 (2011).

47. Stamatakis, A. RAxML version 8: a tool for phylogenetic analysis and postanalysis of large phylogenies. Bioinformatics 30, 1312-1313 (2014). 
48. Hori, K. et al. Klebsormidium flaccidum genome reveals primary factors for plant terrestrial adaptation. Nat. Commun. 5, 3978 (2014).

49. Worden, A. Z. et al. Green evolution and dynamic adaptations revealed by genomes of the marine picoeukaryotes Micromonas. Science 324, 268-272 (2009).

50. Holmgren, A. Thioredoxin structure and mechanism: conformational changes on oxidation of the active-site sulfhydryls to a disulfide. Structure 3, 239-243 (1995).

51. Yu, Q. B. et al. AtECB1/MRL7, a thioredoxin-like fold protein with disulfide reductase activity, regulates chloroplast gene expression and chloroplast biogenesis in Arabidopsis thaliana. Mol. Plant 7, 206-217 (2014).

52. Katti, S. K., LeMaster, D. M. \& Eklund, H. Crystal structure of thioredoxin from Escherichia coli at 1.68 A resolution. J. Mol. Biol. 212, 167-184 (1990).

53. Holmgren, A. Thioredoxin catalyzes the reduction of insulin disulfides by dithiothreitol and dihydrolipoamide. J. Biol. Chem. 254, 9627-9632 (1979).

54. Qiu, Y. et al. HEMERA couples the proteolysis and transcriptional activity of PHYTOCHROME INTERACTING FACTORs in Arabidopsis photomorphogenesis. Plant Cell 27, 1409-1427 (2015).

55. Galvao, R. M. et al. Photoactivated phytochromes interact with HEMERA and promote its accumulation to establish photomorphogenesis in Arabidopsis. Genes Dev. 26, 1851-1863 (2012).

56. Ghosh, S., Hamdan, S. M., Cook, T. E. \& Richardson, C. C. Interactions of Escherichia coli thioredoxin, the processivity factor, with bacteriophage T7 DNA polymerase and helicase. J. Biol. Chem. 283, 32077-32084 (2008).

57. Huber, H. E., Russel, M., Model, P. \& Richardson, C. C. Interaction of mutant thioredoxins of Escherichia coli with the gene 5 protein of phage T7. The redox capacity of thioredoxin is not required for stimulation of DNA polymerase activity. J. Biol. Chem. 261, 15006-15012 (1986).

58. Akabayov, B. et al. Conformational dynamics of bacteriophage T7 DNA polymerase and its processivity factor, Escherichia coli thioredoxin. Proc. Natl Acad. Sci. USA 107, 15033-15038 (2010).

59. Rosso, M. G. et al. An Arabidopsis thaliana T-DNA mutagenized population (GABI-Kat) for flanking sequence tag-based reverse genetics. Plant Mol. Biol. 53, 247-259 (2003)

60. Mirouze, M. et al. Selective epigenetic control of retrotransposition in Arabidopsis. Nature 461, 427-430 (2009).

61. Cao, J. et al. Whole-genome sequencing of multiple Arabidopsis thaliana populations. Nat. Genet. 43, 956-963 (2011).

62. Laitinen, R. A. E., Schneeberger, K., Jelly, N. S., Ossowski, S. \& Weigel, D. Identification of a spontaneous frame shift mutation in a nonreference Arabidopsis accession using whole genome sequencing. Plant Physiol. 153, 652-654 (2010).

63. Schneeberger, K. et al. SHOREmap: simultaneous mapping and mutation identification by deep sequencing. Nat. Methods 6, 550-551 (2009).

64. Fankhauser, C. et al. PKS1, a substrate phosphorylated by phytochrome that modulates light signaling in Arabidopsis. Science 284, 1539-1541 (1999).

65. Goodstein, D. M. et al. Phytozome: a comparative platform for green plant genomics. Nucleic Acids Res. 40, D1178-D1186 (2012).

66. Sundell, D. et al. The plant genome integrative explorer resource: PlantGenIE. org. New Phytol. 208, 1149-1156 (2015).

67. Matasci, N. et al. Data access for the 1,000 Plants (1KP) project. Gigascience 3, 17 (2014).

68. Li, F.-W. et al. Horizontal transfer of an adaptive chimeric photoreceptor from bryophytes to ferns. Proc. Natl Acad. Sci. USA 111, 6672-6677 (2014).

69. Amborella Genome Project. The Amborella genome and the evolution of flowering plants. Science 342, 1241089 (2013).

70. Edgar, R. C. MUSCLE: a multiple sequence alignment method with reduced time and space complexity. BMC Bioinforma. 5, 113 (2004).

71. Lanfear, R., Calcott, B., Ho, S. Y. W. \& Guindon, S. Partitionfinder: combined selection of partitioning schemes and substitution models for phylogenetic analyses. Mol. Biol. Evol. 29, 1695-1701 (2012).

72. Chi-Ham, C. L., Keaton, M. A., Cannon, G. C. \& Heinhorst, S. The DNAcompacting protein DCP68 from soybean chloroplasts is ferredoxin:sulfite reductase and co-localizes with the organellar nucleoid. Plant Mol. Biol. 49, 621-631 (2002).

73. Wojtaszek, J. et al. Multifaceted recognition of vertebrate Rev1 by translesion polymerases $\zeta$ and к. J. Biol. Chem. 287, 26400-26408 (2012).
74. Delaglio, F. et al. NMRPipe: a multidimensional spectral processing system based on UNIX pipes. J. Biomol. NMR 6, 277-293 (1995).

75. Goddard, T. D. \& Kneller, D. G. SPARKY 3. (University of California, San Francisco, 2008).

76. Shen, Y., Delaglio, F., Cornilescu, G. \& Bax, A. TALOS+: a hybrid method for predicting protein backbone torsion angles from NMR chemical shifts. J. Biomol. NMR 44, 213-223 (2009).

77. Güntert, P. Automated NMR structure calculation with CYANA. Methods Mol. Biol. 278, 353-378 (2004).

78. Chen, V. B. et al. MolProbity: all-atom structure validation for macromolecular crystallography. Acta Crystallogr. D. Biol. Crystallogr. 66, 12-21 (2010).

79. Pfalz, J. Investigating the assembly status of the plastid encoded polymerase using BN-PAGE and sucrose gradient centrifugation. Bio-protocol. 6, 1873 (2016)

\section{Acknowledgements}

We thank Akira Nagatani for providing the $P B G$ line, Clark Lagarias for the $Y H B$ line, and Peter Quail for the pifq mutant, Sabine Heinhorst for anti-SiR antibodies. We thank Yongjian Qiu and Elise Pasoreck for helpful discussions and assistance with the paper. We thank Charles Delwiche and Caren Chang for valuable suggestions regarding the phylogenetic analysis of NCP and RCB. We thank Joanne Chory and Sabeeha Merchant for suggestions about naming of the $n c p$ mutant. This work was supported by the National Institute of General Medical Sciences grant R01GM087388 and the National Science Foundation grant IOS-1051602 to M.C.

\section{Author contributions}

E.J.Y., C.Y., J.L., P.Z. and M.C. conceived the original research plan. M.C., P.Z., T.P., D.W. and K.M.P. supervised the experiments. E.J.Y., C.Y., J.L., H.W. and J.C. performed the experiments. E.J.Y., C.Y., J.L., H.W., J.C., P.Z. and M.C. analyzed the data. J.C. and D.W. carried out the SHOREmap experiments. E.J.Y., F.L. and K.M.P. performed the phylogenetic analysis. E.J.Y., C.Y., P.Z. and M.C. wrote the article with contributions from all the authors.

\section{Additional information}

Supplementary Information accompanies this paper at https://doi.org/10.1038/s41467019-10517-1.

Competing interests: The authors declare no competing interests.

Reprints and permission information is available online at http://npg.nature.com/ reprintsandpermissions/

Peer review information: Nature Communications thanks Alexander Shekhtman and other anonymous reviewer(s) for their contribution to the peer review of this work. Peer reviewer reports are available.

Publisher's note: Springer Nature remains neutral with regard to jurisdictional claims in published maps and institutional affiliations.

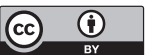

Open Access This article is licensed under a Creative Commons Attribution 4.0 International License, which permits use, sharing, adaptation, distribution and reproduction in any medium or format, as long as you give appropriate credit to the original author(s) and the source, provide a link to the Creative Commons license, and indicate if changes were made. The images or other third party material in this article are included in the article's Creative Commons license, unless indicated otherwise in a credit line to the material. If material is not included in the article's Creative Commons license and your intended use is not permitted by statutory regulation or exceeds the permitted use, you will need to obtain permission directly from the copyright holder. To view a copy of this license, visit http://creativecommons.org/ licenses/by/4.0/

(C) The Author(s) 2019 